\title{
Generic-reference and generic-generic bioequivalence of forty-two, randomly- selected, on-market generic products of fourteen immediate-release oral drugs
}

Muhammad M. Hammamii ${ }^{1,2^{*}}$, Sophia J. S. De Padua ${ }^{1}$, Rajaa Hussein ${ }^{1}$, Eman Al Gaai ${ }^{1}$, Nesrine A. Khodr ${ }^{1}$, Reem Al-Swayeh', Syed N. Alvi ${ }^{1}$ and Nada Binhashim ${ }^{1}$

\begin{abstract}
Background: The extents of generic-reference and generic-generic average bioequivalence and intra-subject variation of on-market drug products have not been prospectively studied on a large scale.

Methods: We assessed bioequivalence of 42 generic products of 14 immediate-release oral drugs with the highest number of generic products on the Saudi market. We conducted 14 four-sequence, randomized, crossover studies on the reference and three randomly-selected generic products of amlodipine, amoxicillin, atenolol, cephalexin, ciprofloxacin, clarithromycin, diclofenac, ibuprofen, fluconazole, metformin, metronidazole, paracetamol, omeprazole, and ranitidine. Geometric mean ratios of maximum concentration $\left(C_{\max }\right)$ and area-under-the-concentration-time-curve, to last measured concentration $\left(A \cup C_{T}\right)$, extrapolated to infinity $\left(A \cup C_{1}\right)$, or truncated to $C_{\max }$ time of reference product ( $A \cup C_{\text {Reftmax }}$ ) were calculated using non-compartmental method and their 90\% confidence intervals (Cl) were compared to the 80.00\%-125. $00 \%$ bioequivalence range. Percentages of individual ratios falling outside the $\pm 25 \%$ range were also determined.
\end{abstract}

Results: Mean (SD) age and body-mass-index of 700 healthy volunteers (28-80/study) were 32.2 (6.2) years and 24.4 (3.2) $\mathrm{kg} / \mathrm{m}^{2}$, respectively. In 42 generic-reference comparisons, 100\% of AUC $C_{T}$ and $A \cup C_{1}$ Cls showed bioequivalence, $9.5 \%$ of $C_{\max }$ Cls barely failed to show bioequivalence, and $66.7 \%$ of $A \cup C_{\text {Reftmax }}$ Cls failed to show bioequivalence/showed bioinequivalence. Adjusting for 6 comparisons, 2.4\% of $A \cup C_{T}$ and $A \cup C_{1} C_{1}$ and 21.4\% of $C_{\max } C_{l s}$ failed to show bioequivalence. In 42 generic-generic comparisons, 2.4\% of $A \cup C_{T}$, $A \cup C_{1}$, and $C_{\max }$ Cls failed to show bioequivalence, and $66.7 \%$ of $A \cup C_{\text {Reftmax }}$ Cls failed to show bioequivalence/showed bioinequivalence. Adjusting for 6 comparisons, 2. $4 \%$ of $A \cup C_{T}$ and $A \cup C_{1} C_{l}$ and $14.3 \%$ of $C_{\max }$ Cls failed to show bioequivalence. Average geometric mean ratio deviation from $100 \%$ was $\leq 3.2$ and $\leq 5.4$ percentage points for $A \cup C_{1}$ and $C_{\text {max }}$ respectively, in both generic-reference and genericgeneric comparisons. Individual generic/reference and generic/generic ratios, respectively, were within the $\pm 25 \%$ range in $>75 \%$ of individuals in $79 \%$ and $71 \%$ of the 14 drugs for $A U C_{T}$ and $36 \%$ and $29 \%$ for $C_{\max }$.

Conclusions: On-market generic drug products continue to be reference-bioequivalent and are bioequivalent to each other based on $A \cup C_{T}, A \cup C_{1}$, and $C_{\max }$ but not $A \cup C_{\text {Reftmax }}$. Average deviation of geometric mean ratios and intra-subject variations are similar between reference-generic and generic-generic comparisons.

Trial registration: ClinicalTrials.gov identifier: NCT01344070 (registered April 3, 2011).

\footnotetext{
* Correspondence: Muhammad@kfshrc.edu.sa

${ }^{1}$ Clinical Studies and Empirical Ethics Department, King Faisal Specialist

Hospital and Research Center, P O Box \# 3354, MBC 03, Riyadh 11211, Saudi

Arabia

${ }^{2}$ Alfaisal University College of Medicine, Riyadh, Saudi Arabia
} 


\section{Background}

One of the causes of economic inefficiency in healthcare is underuse of generic drug products [1], which is due, in part, to mistrust by healthcare professionals [2] and patients [3] and may be related to information availability [4], educational level [3], and healthcare system maturity $[2,5,6]$.

An application for marketing approval of a generic drug product must provide evidence of its bioequivalence $(\mathrm{BE})$ to a reference product that was approved based on clinical trials [7-9]. Although there are some differences among regulatory agencies worldwide [7-9], for immediate-release drugs, average bioequivalence (BE) testing is commonly performed in a single-dose, crossover study on healthy volunteers under fasting condition; with measurement of parent drug blood concentration, non-compartmental analysis of logarithmically transformed area-under-the-concentration-time curve (AUC) and maximum concentration $\left(\mathrm{C}_{\max }\right)$ data, and computation of the $90 \%$ confidence interval (CI) on the test/reference geometric mean ratio, which should generally fall within the $80-125 \% \mathrm{BE}$ range $[10,11]$.

Establishing surveillance systems of on-market generic products has been advocated [4] because of sporadic concerns about post-marketing quality [12-16]. Although several clinical studies [17-21] failed to detect important differences between reference and generic products, direct bioequivalence studies are limited [16, 17, 22].

Under current regulations, BE studies among onmarket, reference-bioequivalent, generic products are not required, which raises the theoretical concern that a generic product at one end of the $\mathrm{BE}$ range might not be equivalent to another at the other end [23-25]. Few studies have addressed the issue; using retrospective analysis of reference-normalized data [26-28], simulation $[29,30]$, or a prospective but restricted approach [31].

One size-fits-all BE approach may not adequately take intra-subject variability and therapeutic windows into account [32-34]. Intra-subject variability can be due to intra-drug variability (physiological metabolic variability), intra-product variability (unit to unit or batch to batch), or subject-by-product interaction. Generic intra-product variability and subject-by-product interaction are especially important for narrow therapeutic index (NTI) drugs, for which the $75 / 75$ rule $(75 \%$ of individual ratios are within $\pm 25 \%$ ), among other methods of analysis, have been proposed [10, 35]. A simulation study was assuring [25] and few studies specific to antiepileptic medications [17, 28, 31] provided further support of the applicability of current BE standards to NTI drugs and led to revision of the American Epilepsy Society's guidelines concerning reference-to-generic and generic-to-generic switching [36]. However, there are still concerns that the results may not apply to countries with less stringent control over pharmaceuticals' quality [37].
In Saudi Arabia, the Saudi FDA requires demonstration of BE (applying the 80.00-125.00\% BE limits on $\mathrm{C}_{\max }$ and AUC 90\% CIs) before registering generic drug products, registered products are listed in the Saudi National Formulary, generic substitution for none-NTI drugs by pharmacists is permissive with patient's consent, and generic prescribing is encouraged [38]. Although the Saudi FDA has a policy to reexamine the products for which it receives complaints, it does not systemically assess the $\mathrm{BE}$ of on-market generic products. A 2015 study on a random sample of 178 physicians in 2 hospitals in the Riyadh showed that although $52 \%$ supported substitution by local generic products, only $22 \%$ believed that Saudi FDA-approved, local generic products are therapeutically equivalent to reference products [39].

Given the tremendous cost-saving and potential improvement in healthcare accessibility provided by generic drug products, the serious clinical implications of prescribing products with unacceptable bioavailability or switching between products that are not bioequivalent, the need to alleviate patients and healthcare professionals mistrust, and the paucity of empirical data world-wide, we set the present study as a field test of the current BE standards. Our main aim was to determine the extent of $\mathrm{BE}$ between on-market generic and reference products and among referencebioequivalent generic products. We also examined the percentages of individual, generic/reference and generic/ generic, pharmacokinetic parameters ratios that are outside the $\pm 25 \%$ range.

\section{Methods}

Design

We identified the 15 oral, immediate-release, noncombinational drugs with the highest number of generic products on the Saudi National Formulary. We studied 14 out of the 15 drugs because the reference (R) product of one of them (enalapril) was not available on the Saudi market. On each drug, we conducted four-product, foursequence, four-period, sequence-randomized, crossover $\mathrm{BE}$ study using the $\mathrm{R}$ product and 3 randomly-selected generic products $(\mathrm{Ga}, \mathrm{Gb}$, and $\mathrm{Gc})$. The four sequences, namely, Ga-Gb-Gc-R, Gb-R-Ga-Gc, Gc-Ga-R-Gb, and R-Gc-Gb-Ga, were designed so that every product appears the same number of time within each period and each sequence, and every product follows every other product the same number of times. Washout periods and blood sampling frames were drug-specific (Table 1) and extended to about 7 and 5 drug plasma half-lives, respectively.

\section{Participants}

We enrolled healthy, non-pregnant adults (age 18-60 years) with a body mass index (BMI) $\leq 35 \mathrm{~kg} / \mathrm{m}^{2}$, who accepted to 
Table 1 Summary of fourteen 4-product, 4-sequence, 4-period, sequence-randomized, crossover bioequivalence studies on 14 immediate-release, non-combinational, oral drugs

\begin{tabular}{|c|c|c|c|c|c|c|c|c|c|}
\hline Drug & $\begin{array}{l}\text { Participants, } \\
\text { no., sex }\end{array}$ & $\begin{array}{l}\text { Age, } \\
\text { mean } \\
\text { (SD), } \\
\text { year }\end{array}$ & $\begin{array}{l}\mathrm{BMl}, \\
\text { mean } \\
(\mathrm{SD}), \\
\mathrm{kg} / \mathrm{m}^{2}\end{array}$ & $\begin{array}{l}\text { Washout } \\
\text { period, } \\
\text { day }\end{array}$ & $\begin{array}{l}\text { Sampling frame, } \\
\text { hour }\end{array}$ & $\begin{array}{l}\text { Withdrawals, no. } \\
\text { (no. missed } \\
\text { periods, reason) }\end{array}$ & $\begin{array}{l}\text { Possible } \\
\text { product } \\
\text { failure, no. } \\
\text { (product, period) }\end{array}$ & $\begin{array}{l}\text { Adverse } \\
\text { events (no.) }\end{array}$ & $\begin{array}{l}\text { Assay } \\
\text { (lower } \\
\text { quantification limit) }\end{array}$ \\
\hline $\begin{array}{l}\text { Amlodipine } \\
10 \mathrm{mg}\end{array}$ & $\begin{array}{l}54 \mathrm{M} \\
2 \mathrm{~F}\end{array}$ & $\begin{array}{l}34.0 \\
(7.2)\end{array}$ & $\begin{array}{l}24.3 \\
(3.0)\end{array}$ & 14 & 240 & $\begin{array}{l}1(1, \text { venous access }) \\
1(4, \text { personal })\end{array}$ & 1 (reference, 3rd) & $\begin{array}{l}\text { Headache (1) } \\
\text { Drowsiness (1) }\end{array}$ & $\begin{array}{l}\text { LC-MS } \\
(0.20 \mathrm{ng} / \mathrm{ml})\end{array}$ \\
\hline $\begin{array}{l}\text { Amoxicillin } \\
500\end{array}$ & $52 \mathrm{M}$ & $\begin{array}{l}31.2 \\
(4.5)\end{array}$ & $\begin{array}{l}24.2 \\
(2.8)\end{array}$ & $3-7$ & 10 & $3(3$, personal) & None & Dizziness (1) & $\begin{array}{l}\text { HPLC } \\
(0.50 \mu \mathrm{g} / \mathrm{ml})\end{array}$ \\
\hline $\begin{array}{l}\text { Atenolol } \\
100 \mathrm{mg}\end{array}$ & $52 \mathrm{M}$ & $\begin{array}{l}30.5 \\
(5.0)\end{array}$ & $\begin{array}{l}23.0 \\
(2.3)\end{array}$ & 7 & 36 & $\begin{array}{l}2 \text { ( } 3 \text {, Flu-like symptoms) } \\
2 \text { (4, personal) }\end{array}$ & None & $\begin{array}{l}\text { Flu-like symptoms } \\
\text { (2) } \\
\text { Vomiting (1) }\end{array}$ & $\begin{array}{l}\text { HPLC } \\
(0.01 \mu \mathrm{g} / \mathrm{ml}\end{array}$ \\
\hline $\begin{array}{l}\text { Cephalexin } \\
500 \mathrm{mg}\end{array}$ & $36 \mathrm{M}$ & $\begin{array}{l}32.3 \\
(7.3)\end{array}$ & $\begin{array}{l}24.5 \\
(5.0)\end{array}$ & $2-7$ & 6 & 4 (3, personal) & None & Headache (1) & $\begin{array}{l}\text { HPLC } \\
(0.50 \mu \mathrm{g} / \mathrm{ml})\end{array}$ \\
\hline $\begin{array}{l}\text { Ciprofloxacin } \\
500 \mathrm{mg}\end{array}$ & $44 \mathrm{M}$ & $\begin{array}{l}34.6 \\
(6.5)\end{array}$ & $\begin{array}{l}26.1 \\
(3.7)\end{array}$ & 7 & 24 & $\begin{array}{l}1 \text { (2, personal) } \\
1 \text { (3, skin rash) } \\
1(4, \text { high BP })\end{array}$ & None & Skin rash (1) & $\begin{array}{l}\text { HPLC } \\
(0.10 \mu \mathrm{g} / \mathrm{ml})\end{array}$ \\
\hline $\begin{array}{l}\text { Clarithromycin } \\
500 \mathrm{mg}\end{array}$ & $48 \mathrm{M}$ & $\begin{array}{l}30.8 \\
(5.0)\end{array}$ & $\begin{array}{l}23.5 \\
(2.6)\end{array}$ & 7 & 24 & 1 (1, venous access) & None & $\begin{array}{l}\text { Headache (1) } \\
\text { Stomach upset (1) }\end{array}$ & $\begin{array}{l}\text { LC-MS } \\
(5.0 \mathrm{ng} / \mathrm{ml})\end{array}$ \\
\hline $\begin{array}{l}\text { Diclofenac } \\
50 \mathrm{mg}\end{array}$ & $72 \mathrm{M}$ & $\begin{array}{l}30.9 \\
(5.4)\end{array}$ & $\begin{array}{l}24.0 \\
(3.0)\end{array}$ & $2-7$ & 6 & $\begin{array}{l}2(1, \text { personal }) \\
1(1, \text { incompliance }) \\
2(3, \text { personal) }\end{array}$ & None & $\begin{array}{l}\text { Dizziness (1) } \\
\text { Cough (1) }\end{array}$ & $\begin{array}{l}\text { HPLC } \\
(0.02 \mu \mathrm{g} / \mathrm{ml})\end{array}$ \\
\hline $\begin{array}{l}\text { Ibuprofen } \\
400 \mathrm{mg}\end{array}$ & $\begin{array}{l}30 \mathrm{M} \\
2 \mathrm{~F}\end{array}$ & $\begin{array}{l}34.6 \\
(9.0)\end{array}$ & $\begin{array}{l}25.6 \\
(3.3)\end{array}$ & 7 & 10 & $\begin{array}{l}1 \text { (1, personal) } \\
1 \text { ( } 2, \text { personal) } \\
4(3, \text { personal })\end{array}$ & 1 (reference, 2nd) & Near fainting (1) & $\begin{array}{l}\text { HPLC } \\
(0.25 \mu \mathrm{g} / \mathrm{ml})\end{array}$ \\
\hline $\begin{array}{l}\text { Fluconazole } \\
150 \mathrm{mg}\end{array}$ & $28 \mathrm{M}$ & $\begin{array}{l}36.9 \\
(8.7)\end{array}$ & $\begin{array}{l}24.4 \\
(3.0)\end{array}$ & 14 & 168 & $\begin{array}{l}1 \text { (2, skin rash }) \\
2(4, \text { personal })\end{array}$ & None & $\begin{array}{l}\text { Skin rash (1) } \\
\text { Headache (1) }\end{array}$ & $\begin{array}{l}\text { HPLC } \\
(0.20 \mu \mathrm{g} / \mathrm{ml})\end{array}$ \\
\hline $\begin{array}{l}\text { Metformin } \\
850 \mathrm{mg}\end{array}$ & $52 \mathrm{M}$ & $\begin{array}{l}31.9 \\
(5.8)\end{array}$ & $\begin{array}{l}23.9 \\
(2.6)\end{array}$ & 7 & 32 & $\begin{array}{l}1(1, \text { personal }) \\
1(2, \text { personal }) \\
1(3, \text { personal }) \\
1(4, \text { personal })\end{array}$ & None & $\begin{array}{l}\text { Diarrhea (1) } \\
\text { Headache (1) }\end{array}$ & $\begin{array}{l}\text { HPLC } \\
(0.05 \mu \mathrm{g} / \mathrm{ml})\end{array}$ \\
\hline $\begin{array}{l}\text { Metronidazole } \\
250 \mathrm{mg}\end{array}$ & $28 \mathrm{M}$ & $\begin{array}{l}31.8 \\
(5.6)\end{array}$ & $\begin{array}{l}24.3 \\
(2.8)\end{array}$ & 7 & 48 & None & 1 (generic b, 1st) & Headache (2) & $\begin{array}{l}\text { HPLC } \\
(0.05 \mu \mathrm{g} / \mathrm{ml})\end{array}$ \\
\hline $\begin{array}{l}\text { Omeprazole } \\
20 \mathrm{mg}\end{array}$ & $80 \mathrm{M}$ & $\begin{array}{l}31.8 \\
(5.0)\end{array}$ & $\begin{array}{l}24.8 \\
(3.5)\end{array}$ & 7 & 12 & $\begin{array}{l}1(1, \text { personal }) \\
1(2, \text { personal }) \\
3(3 \text {, personal) } \\
1(4 \text {, incompliance }) \\
1(4, \text { high BP })\end{array}$ & None & Dizziness (2) & $\begin{array}{l}\text { HPLC } \\
(0.01 \mu \mathrm{g} / \mathrm{ml})\end{array}$ \\
\hline $\begin{array}{l}\text { Paracetamol } \\
500 \mathrm{mg}\end{array}$ & $44 \mathrm{M}$ & $\begin{array}{l}32.3 \\
(6.2)\end{array}$ & $\begin{array}{l}24.1 \\
(3.6)\end{array}$ & $2-7$ & 14 & $\begin{array}{l}1 \text { (2, personal) } \\
3(3, \text { personal) } \\
1(4 \text {, incompliance })\end{array}$ & $\begin{array}{l}1 \text { (generic b, } \\
\text { 2nd) }\end{array}$ & None & $\begin{array}{l}\text { HPLC } \\
(0.10 \mu \mathrm{g} / \mathrm{ml})\end{array}$ \\
\hline $\begin{array}{l}\text { Ranitidine } \\
150 \mathrm{mg}\end{array}$ & $\begin{array}{l}74 \mathrm{M} \\
2 \mathrm{~F}\end{array}$ & $\begin{array}{l}31.8 \\
(5.5)\end{array}$ & $\begin{array}{l}25.2 \\
(3.2)\end{array}$ & $2-7$ & 14 & $\begin{array}{l}1 \text { (1, personal) } \\
1 \text { (2, venous access) } \\
1 \text { ( } 3 \text {, venous access }) \\
1 \text { ( } 3 \text { incompliance }) \\
1 \text { ( } 3 \text {, vomiting) } \\
1 \text { ( } 4 \text {, venous access) }\end{array}$ & None & $\begin{array}{l}\text { Vomiting (2) } \\
\text { Diarrhea (2) } \\
\text { Dizziness (1) }\end{array}$ & $\begin{array}{l}\text { HPLC } \\
(0.03 \mu \mathrm{g} / \mathrm{ml})\end{array}$ \\
\hline
\end{tabular}

Eighteen blood samples were obtained during each period of each study

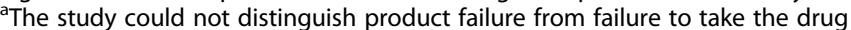

${ }^{\mathrm{b}}$ All adverse events were minor and resolved spontaneously. HPLC High performance liquid chromatography, LC-MS Liquid chromatography-mass spectrometry, BP Blood pressure. Flu-like, influenza-like

abstain from taking any medication for $\geq 2$ weeks before, and during the study, and from smoking, alcohol, and xanthene-containing beverages or food for $\geq 48 \mathrm{~h}$ before, and during each of the four study periods. Volunteers were screened by medical history, physical exam, and laboratory tests that included complete blood count, renal profile, and liver profile. Subjects with history of hypersensitivity to the drug to be tested, recent acute illness, or clinicallyimportant laboratory tests' abnormality were excluded. For menstruating women, the study was conducted 5 to 19 days after last menstrual period and after obtaining a negative urine pregnancy test.

The study was conducted at the King Faisal Specialist Hospital \& Research Center (KFSH\&RC), Riyadh from May 2011 through April 2015 in accordance with Declaration of Helsinki ethics principles and good clinical practice and after obtaining approval of the KFSH\&RC Research Ethics Committee. Each participant gave written informed 
consent at enrolment and was compensated based on the Wage-Payment model [40] in a prorated manner.

\section{Procedures and interventions}

Reference and generic drug products were purchased from retail pharmacies in Riyadh, Saudi Arabia.

After fasting for $10 \mathrm{~h}$, drug products were administered with $240 \mathrm{ml}$ of water at room temperature. Fasting from food and beverages continued for $4 \mathrm{~h}$ post-dosing. However, volunteers were allowed $120 \mathrm{ml}$ water every hour, except for $1 \mathrm{~h}$ before and $1 \mathrm{~h}$ after drug administration. Standardized breakfast and standardized dinner were given 4 and $10 \mathrm{~h}$ after drug administration. Meal plans were identical in the four study periods. Volunteers remained ambulatory or seated upright (unless deemed medically necessary) for $4 \mathrm{~h}$ after drug administration. Strenuous physical activity was not permitted during study periods.

During each study period, in addition to a baseline blood sample, 17 blood samples were drawn (Additional file 1). Sampling schedules were drug specific and were designed to collect adequate number of samples before and around the expected $C_{\max }$ and across 5 half-lives of the drug. Blood samples were collected in vacutainer tubes and centrifuged for $10 \mathrm{~min}$ at room temperature within $15 \mathrm{~min}$ of collection. Plasma samples were harvested in clean polypropylene tubes and placed immediately at $-80^{\circ} \mathrm{C}$ until analysed.

Compliance with study protocol was checked before drug administration in each study period. Volunteers were under continuous observation regarding occurrence of adverse events and compliance with study protocol during the first day of each period. In addition, they were asked about experiencing adverse events at the time of last blood collection of each period and at the beginning of subsequent periods.

Drug concentrations were blindly measured by inhouse, locally-validated, reversed-phase high performance liquid chromatography (HPLC) [41-52] or liquid chromatography-mass spectrometry (LC-MS) [53, 54]. Lower limits of quantification are listed in Table 1. Intra-assay coefficient of variation (standard deviation/ mean *100) and bias (measured concentration/nominal concentration * 100) were $\leq 3.1-14.4$ and $\leq 5.0-17.0$, respectively. A typical assay run included a series of 10 calibrators and several sets of four quality control samples ( 1 and 3 times lower quantification limit and 0.5 and $0.8-0.9$ upper quantification limit). Samples from the four periods for each volunteer were analyzed in the same run. Samples with drug concentration greater than the upper quantification limit were reassayed after dilution. Samples with drug concentration below the lower quantification limit were assigned zero concentration. Drug concentrations of missing samples were assigned the average concentration of the two flanking samples in the same period.

\section{Random sampling of generic drug products and randomization}

For each of the 14 drugs, all of the Saudi formulary-listed generic products were assigned sequential numbers, the numbers were arranged randomly (by $\mathrm{MMH}$ ) using an online random number generator [55], and the three generic products corresponding to the first three randomly-arranged numbers were selected and labeled $\mathrm{Ga}, \mathrm{Gb}$, and $\mathrm{Gc}$, respectively.

For each of the 14 studies, blocked (block size $=4$ ) randomization sequences were generated (by $\mathrm{MMH}$ ) using an online program [55]. Randomization sequences were concealed from recruiting study coordinators and from potential participants.

\section{Sample size}

Sample size for each study was estimated using an online program [56]; assuming an $\mathrm{AUC}_{\mathrm{I}}$ and $\mathrm{C}_{\max }$ ratio of generic to reference product of 1.10 , a power of 0.9 , a left equivalence limit of 0.80 , a right equivalence limit of 1.25 , and 2 one-sided type I error of 0.05 , Bonferroniadjusted for 6 comparisons (i.e., $\alpha=0.0083$ ). Sample size was rounded and inflated by 3-8 subjects to allow for potential withdrawals/dropouts. Intra-subject coefficient of variation $(\mathrm{CV})$ was estimated from published studies as $50 \%$ of reported total CV (Additional file 2).

\section{Outcome measures and analysis}

The following pharmacokinetic parameters were determined using standard non-compartmental methods: $\mathrm{AUC}_{\mathrm{T}}$ (area-under-the-concentration-time curve from time zero to time of last measured concentration) calculated by linear trapezoidal method, $\mathrm{AUC}_{\mathrm{I}}$ (area-under-the-concentrationtime curve from time 0 to infinity) calculated as $\mathrm{AUC}_{\mathrm{T}}$ plus the ratio of last measured concentration to elimination rate constant, $\mathrm{AUC}_{\mathrm{T}} / \mathrm{AUC}_{\mathrm{I}}, \mathrm{C}_{\max }$ (maximum concentration) determined directly from the observed data, $\mathrm{T}_{\max }$ (first time of maximum concentration) determined directly from the observed data, $\lambda$ (apparent first-order elimination rate constant) calculated by linear least-squares regression analysis from the last 4-8 quantifiable concentrations of a plot of natural log-transformed concentration versus time curve, $t_{1 / 2}$ (terminal elimination half-life) calculated as $\ln 2 /$ $\lambda, \mathrm{AUC}_{72}$ (area-under-the-concentration-time curve truncated to $72 \mathrm{~h}$ ) calculated by linear trapezoidal method, and AUC $_{\text {Reftmax }}$ (area-under-the-concentration-time curve to $\mathrm{T}_{\max }$ of reference product, calculated for each subject) calculated by linear trapezoidal method. When $\lambda$ was not calculable in a given study period, the average of $\lambda$ s in other periods of the same volunteer was used to calculate $A U C_{I}$ for that period. $A U C_{\text {Reftmax }}$ was not calculated when data 
for the reference product were missing. Each generic $\mathrm{AUC}_{\text {Reftmax }}$ with zero value was assigned 0.001 in order to perform log-transformation. Pharmacokinetic and statistical analyses included all evaluable data of all volunteers.

Primary outcome measures were $\mathrm{C}_{\max }, \mathrm{AUC}_{\mathrm{T}}$, and $\mathrm{AUC}_{\mathrm{I}}$. Secondary outcome measures were $\mathrm{T}_{\max }, \mathrm{AUC}_{\mathrm{Reftmax}}$, and $\mathrm{AUC}_{72}$. The four products of each drug were compared by analysis of variance (ANOVA). The ANOVA model included, product, period, sequence, and subjects nested in sequence. Mean square residual (MSR) was used to test significance of period and product effects. Subjects nested in sequence mean square was used to test significance of sequence effect. For each pharmacokinetic parameter (except $\mathrm{T}_{\max }$ ), six pairwise (Ga-R, Gb-R, Gc-R, Ga-Gb, GbGc, and Ga-Gc) $90 \%$ CIs on the difference between means of log-transformed values (i.e., geometric mean ratio) were determined using MSR without and with Bonferroni adjustment for 3 or 6 comparisons, and the antilogs of the $90 \% \mathrm{CI}$ limits were compared to the BE limits of $80.00 \%$ and $125.00 \%$. The null hypothesis (lack of bioequivalence) was rejected if the $90 \%$ CI was completely within $80.00 \%$ to $125.00 \%$. If the null hypothesis was not rejected, the analysis would indicate either failure to show bioequivalence (the 90\% CI crosses the BE limits) or bioinequivalence (the 90\% $\mathrm{CI}$ is completely outside the $\mathrm{BE}$ limits). to The following were also calculated: percentage of generic products that are not bioequivalent to their reference product or not bioequivalent to each other based on $C_{\max }, \mathrm{AUC}_{\mathrm{T}}, \mathrm{AUC}_{\mathrm{I}}$, or $\mathrm{AUC} \mathrm{C}_{\text {Reft- }}$ max, mean (SD) deviation of $\mathrm{AUC}_{\mathrm{T}}, \mathrm{AUC}_{\mathrm{I}}$, and $\mathrm{C}_{\mathrm{max}}$ genericreference and generic-generic point estimates from 100\% and percentages of the deviations that were $<6,<10$, or $>13$ percentage points, percentage of individual $C_{\max }, \mathrm{AUC}_{\mathrm{T}}$, $A U C_{I}, A C_{72}, T_{\text {max }}$, and $\mathrm{AUC}_{\text {Reftmax }}$ generic/reference and generic/generic ratios that are $75 \%$ or $125 \%$, and percentage of drugs that failed to fulfil the $75 / 75$ rule (i.e.,75\% of individual ratios are within $\pm 25 \%$ ) for each of the pharmacokinetic parameters. Pharmacokinetic and statistical analyses were performed (by $\mathrm{MMH}$ ) on a personal computer using Microsoft Excel (Version 2010) with add-ins (PK Functions for Microsoft Excel, JI Usansky, A Desai, and D Tang-liu, Department of pharmacokinetics and Drug Metabolism, Allergan Irvine, CA, USA) and IBM SPSS Statistics version 21 software, respectively.

\section{Results}

The 14 immediate-release, non-combinational, oral drugs with the highest number of generic products on the Saudi National Formulary that were assessed were, in descending order, ciprofloxacin (18 generic products), ranitidine, amoxicillin, paracetamol, atenolol, cephalexin, ibuprofen, diclofenac, metformin, omeprazole, metronidazole, clarithromycin, amlodipine, and fluconazole (7 generic products). Commercial name, manufacturer name, formulation, strength, lot/ batch number, manufacture date, and expiry date for the reference and the 3 randomly-selected generic products as well as the number of listed generic products are presented in Additional file 3. About $52 \%$ of the 42 generic products were manufactured in Saudi Arabia, $14 \%$ in other Gulf States, 31\% in Arabic non-Gulf States, and 2\% in Portugal.

Seven hundred healthy volunteers participated in 14, four-product, four-sequence, four-period, sequencerandomized, crossover, BE studies. As shown in Table 1, the number of volunteers per study ranged from 28 to 80 . The volunteers were $100 \%$ males for all but 3 studies which had 3-6\% females. Mean (SD) age ranged from 30.5 (5.0) to 36.9 (8.7) years and mean BMI ranged from $23.0(2.3)$ to $26.1(3.7) \mathrm{kg} / \mathrm{m}^{2}$ per study (grand mean age and BMI 32.2 (6.2) years and $24.4(3.2) \mathrm{kg} / \mathrm{m}^{2}$, respectively). Withdrawal from $\geq$ one period ranged from $0 \%$ to $19 \%$ per drug, with a total of 145 missed periods (out of 2800). Withdrawal reasons were mostly personal but also included inadequate venous access, skin rash, vomiting, high blood pressure, and influenza-like symptoms, as well as incompliance (Table 1). Adverse events occurred in $0 \%$ (paracetamol) to 7\% (fluconazole and metronidazole) of volunteers (Table 1); all were minor and resolved spontaneously.

Baseline drug concentration was not detectable in any period for any of the 14 drugs, indicating adequate wash-out periods. There were 12 missed blood samples ( 2 for clarithromycin, 5 for fluconazole, and 5 for ranitidine) out of the 47,790 scheduled samples (excluding withdrawals); these samples were assigned the average concentration of the two flanking samples of the same volunteer in the same period. In all samples of one volunteer, there was a plasma peak that interfered with the diclofenac assay; this volunteer was excluded from further analysis. In four volunteers, there was no measurable drug concentration in any sample from one study period only (amlodipine, R, 3rd period; ibuprofen, R, 2nd period; metronidazole, Gb, 1st period; and paracetamol, Gb, 2nd period). The unmeasurable concentrations could be due to product failure as the drugs were administered by one of the investigators and the volunteers denied incompliance when confronted; however, incompliance cannot be ruled out. Mean concentrationtime and log-concentration-time curves of the reference and the three generic products of each of the 14 drugs are presented in Additional files 4 and 5, respectively. We were not able to calculated $\lambda$ in a total of $27(1 \%)$ out of the 2647 pharmacokinetic analyses (clarithromycin: (1) Ga, (3) Gb, and (1) Gc; diclofenac: (4) Ga, (4) Gb, (3) Gc, and (7) R; omeprazole: (1) Gb, (2) Gc, and (1) R). Average of $\lambda s$ in other periods of the same volunteer was used to calculate $\mathrm{AUC}_{\mathrm{I}}$ for these 27 analyses. No outlier values for any of the pharmacokinetic parameters were identified or removed from analysis. $\mathrm{AUC}_{\mathrm{T}}$, $\mathrm{AUC}_{\mathrm{I}}, \mathrm{C}_{\max }, \mathrm{T}_{\max }, \lambda, \mathrm{t}_{1 / 2}, \mathrm{C}_{\max } / \mathrm{AUC}_{\mathrm{I}}, \mathrm{AUC}_{\mathrm{T}} / \mathrm{AUC}_{\mathrm{I}}$, 
$A U C_{R e f t m a x}$, and $A C_{72}$ of the reference and the three randomly-selected generic products of each drug are summarized in Additional file 6. $\mathrm{AUC}_{\mathrm{T}} / \mathrm{AUC}_{\mathrm{I}}$ ranged from $90 \%$ (ciprofloxacin) to $98 \%$ (clarithromycin), indicating adequate sampling frames.

MSR from ANOVA analysis and calculated intrasubject $\mathrm{CV}$ for $\mathrm{AUC}_{\mathrm{T}}, \mathrm{AUC}_{\mathrm{I}}$, and $\mathrm{C}_{\max }$ of each drug are presented in Table 2. Significant product, period, and sequence effects on $\mathrm{AUC}_{\mathrm{T}}, \mathrm{AUC}_{\mathrm{I}}$, and $\mathrm{C}_{\max }$ of the 14 drugs are summarized in Additional file 7. MSR and intra-subject $\mathrm{CV}$ for $\mathrm{AUC}_{\text {Reftmax }}$ and $\mathrm{AUC}_{72}$ are presented in Additional files 8 and 9, respectively.

\section{Average bioequivalence of 3 on-market generic products to the reference product of 14 drugs}

Table 2 summarizes the results of the 42 predetermined $\mathrm{BE}$ analyses comparing three randomly-selected generic products to the corresponding reference product of each of the 14 drugs. The results are also depicted in Fig. 1. None of the $\mathrm{AUC}_{\mathrm{T}}$ or $\mathrm{AUC}_{\mathrm{I}} 90 \%$ CIs failed to show bioequivalence and $9.5 \%$ of $\mathrm{C}_{\max } 90 \%$ CIs only barely failed to show bioequivalence. When analyses were adjusted for 3 comparisons, $2.4 \%$ of $\mathrm{AUC}_{\mathrm{T}} 90 \%$ CIs, $0 \%$ of $\mathrm{AUC}_{\mathrm{I}}$ $90 \%$ CIs, and $11.9 \%$ of $\mathrm{C}_{\max } 90 \%$ CIs failed to show bioequivalence, and none showed bioinequivalence. When analyses were adjusted for 6 comparisons, $2.4 \%$ of $\mathrm{AUC}_{\mathrm{T}}$ $90 \%$ CIs (clarithromycin Gc vs. R), $2.4 \%$ of $\mathrm{AUC}_{\mathrm{I}} 90 \%$ CIs (clarithromycin Gc vs. R), and $21.4 \%$ of $C_{\max } 90 \%$ CIs (clarithromycin $\mathrm{Ga}$ and $\mathrm{Gc}$ vs. R; diclofenac $\mathrm{Ga}, \mathrm{Gb}$, and Gc vs. R; ibuprofen Gb and Gc vs. R; omeprazole $\mathrm{Gb}$ and $\mathrm{Gc}$ vs. R) failed to show bioequivalence, and none showed bioinequivalence.

Mean absolute (SD) deviation of point estimates from $100 \%$ in the 42 comparisons was 3.2 (1.8), 3.2 (1.4), and 5.4 (3.3) percentage points for $\mathrm{AUC}_{\mathrm{T}}, \mathrm{AUC}_{\mathrm{I}}$, and $\mathrm{C}_{\text {max }}$, respectively. Further, the deviation was 10 percentage points in 95.2\%, 95.2\%, and $81.0 \%$ of the $\mathrm{AUC}_{\mathrm{T}}, \mathrm{AUC}_{\mathrm{I}}$, and $\mathrm{C}_{\max }$ comparisons, respectively. Furthermore, $0 \%$ of the $\mathrm{AUC}_{\mathrm{T}}$ and $\mathrm{AUC}_{\mathrm{I}}$ and $9.5 \%$ of the $\mathrm{C}_{\max }$ deviations were $>13$ percentage points and $78.6 \%, 81.0 \%$, and $50.0 \%$, respectively, were $<6$ percentage points.

Figure 2 (a) depicts $\mathrm{BE}$ analysis of $\mathrm{AUC}_{\mathrm{Reftmax}}$ between the three generic products and the corresponding reference product of each of the 14 drugs. The data are also summarized in Additional file 8 . Twenty two $(52.4 \%)$ of the $90 \%$ CIs failed to show bioequivalence. In addition, 6 (14.3\%) showed bioinequivalence. Figure 2 (b) depicts $\mathrm{BE}$ analysis of $\mathrm{AUC}_{72}$ between the three generic products and the corresponding reference product of the two drugs with long half-life (amlodipine and fluconazole). BE was demonstrated by all of the six $90 \%$ CIs. The data are also summarized in Additional file 9.
Individual pharmacokinetic parameter ratios of 3 onmarket generic products to the reference product of 14 drugs

There were 1950 individual generic-reference comparisons. The percentages of individual $\mathrm{AUC}_{\mathrm{T}}, \mathrm{AUC}_{\mathrm{I}}$, and $\mathrm{C}_{\max }$, ratios that were outside the $\pm 25 \%$ range are presented in Fig. 3. On average, $16 \%$ of the $\mathrm{AUC}_{\mathrm{T}}$ ratios (ranging from 2\% for cephalexin to 35\% for atenolol and clarithromycin), $15 \%$ of the $\mathrm{AUC}_{\mathrm{I}}$ ratios (ranging from $2 \%$ for cephalexin to $34 \%$ for clarithromycin), and $32 \%$ of $\mathrm{C}_{\max }$ ratios (ranging from $8 \%$ for metronidazole to $57 \%$ for diclofenac), were outside the $\pm 25 \%$ range. Further, individual $\mathrm{AUC}_{\mathrm{T}}, \mathrm{AUC}_{\mathrm{I}}$, and $C_{\max }$, ratios were within the $\pm 25 \%$ range in $75 \%$ of individuals (i.e., fulfilled the $75 / 75$ rule) for $79 \%, 79 \%$, and $36 \%$ of the 14 drugs, respectively.

Out of 161 and $76 \mathrm{AUC}_{72}$ individual ratios for amlodipine and fluconazole, $16 \%$ and $1 \%$, respectively, were outside the $\pm 25 \%$ range (compared to $18 \%$ and $3 \%$, respectively, for $\mathrm{AUC}_{\mathrm{T}}$ ).

Figure 4 depicts the percentages of individual generic/ reference $T_{\max }$ and $A U C_{\text {Reftmax }}$ ratios that were outside the $\pm 25 \%$ range. On average, $60 \%$ of the $T_{\max }$ ratios (ranging from $43 \%$ for amoxicillin to $72 \%$ for ibuprofen) and $58 \%$ of the $\mathrm{AUC}_{\text {Reftmax }}$ ratios (ranging from $27 \%$ for metformin to $89 \%$ for omeprazole) were outside the $\pm 25 \%$ range. Individual $\mathrm{T}_{\max }$ and $\mathrm{AUC}_{\text {Reftmax }}$ ratios were within the $\pm 25 \%$ range in $75 \%$ of individuals for none of the 14 drugs, respectively.

\section{Average bioequivalence among 3 on-market generic products of 14 drugs}

Table 2 also summarizes the results of the 42 predetermined $\mathrm{BE}$ analyses among the three randomly-selected generic products of each of the 14 drugs. The results are also depicted in Fig. 5. Only one (2.4\%) of each of the $\mathrm{AUC}_{\mathrm{T}}, \mathrm{AUC}_{\mathrm{I}}$, and $\mathrm{C}_{\max } 90 \%$ CIs failed to show bioequivalence. When analyses were adjusted for 3 comparisons, $2.4 \%$ of $\mathrm{AUC}_{\mathrm{T}}$ and $\mathrm{AUC}_{\mathrm{I}} 90 \%$ CIs and $9.5 \%$ of $\mathrm{C}_{\max } 90 \%$ CIs failed to show bioequivalence, and none showed bioinequivalence. When analyses were adjusted for 6 comparisons, $2.4 \%$ of $\mathrm{AUC}_{\mathrm{T}}$ and $\mathrm{AUC}_{\mathrm{I}}$ (clarithromycin $\mathrm{Gb}$ vs. Gc) and $14.3 \%$ of $C_{\max } 90 \%$ CIs (cephalexin Ga vs. Gb and $\mathrm{Gb}$ vs. Gc; clarithromycin Gb vs. Gc and Ga vs. Gc; ibuprofen Gb vs. Gc and Ga vs. Gc) failed to show bioequivalence, and none showed bioinequivalence.

Mean absolute (SD) deviation of point estimates from $100 \%$ in the 42 comparisons was 2.5 (2.3), 2.6 (2.2), and 3.3 (3.1) percentage points for $\mathrm{AUC}_{\mathrm{T}}, \mathrm{AUC}_{\mathrm{I}}$, and $\mathrm{C}_{\max }$, respectively. Further, the deviation was $<10$ percentage points in $95.2 \%, 95.2 \%$, and $88.1 \%$ of the $\mathrm{AUC}_{\mathrm{T}}, \mathrm{AUC}_{\mathrm{I}}$, and $\mathrm{C}_{\max }$ comparisons, respectively. Furthermore, only $2.4 \%$ of the $\mathrm{AUC}_{\mathrm{T}}$ and $\mathrm{AUC}_{\mathrm{I}}$ and $7.1 \%$ of the $\mathrm{C}_{\max }$ 
Table 2 Average bioequivalence among 3 randomly-selected generic products and reference product of 14 immediate-release, non-combinational, oral drugs

\begin{tabular}{|c|c|c|c|}
\hline Drug & $A \cup C_{T}$ & $A \cup C_{1}$ & $C_{\max }$ \\
\hline Amlodipine & MSR 0.021, CV 14.6\% & MSR 0.020, CV 14.2\% & MSR 0.027, CV 16.5\% \\
\hline Generic a vs Reference (54) & $98.24 \%(93.76-102.94)$ & $97.84 \%(93.48-102.41)$ & $96.735 \%(91.75-102.00)$ \\
\hline Generic b vs Reference (54) & $96.61 \%(92.20-101.23)$ & 95.83\% (91.56-100.30) & $94.578 \%$ (89.699-99.72) \\
\hline Generic c vs Reference (53) & 98.95\% (94.39-103.72) & 98.14\% (93.72-102.76) & $94.569 \%(89.645-99.76)$ \\
\hline Generic a vs Generic b (55) & 102.34\% (97.71-107.19) & 102.70\% (98.16-107.47) & 101.71\% (96.51-107.19) \\
\hline Generic b vs Generic c (54) & $96.90 \%(92.56-101.63)$ & $97.11 \%(92.78-101.64)$ & $99.84 \%$ (94.69-105.27) \\
\hline Generic a vs Generic c (54) & $99.25 \%(94.72-104.00)$ & $99.76 \%(95.31-104.41)$ & $101.498 \%(96.26-107.02)$ \\
\hline Amoxicillin & MSR 0.012, CV 11.0\% & MSR 0.011, CV $10.5 \%$ & MSR 0.037, CV 19.4\% \\
\hline Generic a vs Reference (49) & $100.68 \%(97.01-104.49)$ & $100.48 \%(96.97-104.12)$ & $98.87 \%(92.63-105.53)$ \\
\hline Generic b vs Reference (49) & $107.45 \%(103.53-111.52)$ & $106.92 \%(103.19-110.79)$ & $109.20 \%(102.30-116.55)$ \\
\hline Generic c vs Reference (49) & 104.99\% (101.16-108.96) & $104.78 \%(101.12-108.58)$ & $111.32 \%(104.29-118.82)$ \\
\hline Generic a vs Generic b (49) & $93.98 \%$ (90.70-97.38) & $93.95 \%(90.67-97.35)$ & $90.54 \%$ (84.83-96.65) \\
\hline Generic b vs Generic c (49) & $101.04 \%(98.48-105.73)$ & 101.93\% (98.36-105.62) & $98.10 \%(91.90-104.71)$ \\
\hline Generic a vs Generic c (49) & $95.89 \%$ (92.55-99.37) & $95.76 \%(92.41-99.23)$ & $88.82 \%$ (83.21-94.80) \\
\hline Atenolol & MSR 0.037, CV 19.4\% & MSR 0.036, CV19.2\% & MSR 0.055, CV 23.8\% \\
\hline Generic a vs Reference (48) & $105.84 \%$ (99.08-113.05) & $105.52 \%(98.88-112.61)$ & $106.46 \%(98.24-115.37)$ \\
\hline Generic b vs Reference (48) & $103.12 \%(96.54-110.14)$ & $102.71 \%(96.25-109.61)$ & 103.10\% (95.14-111.73) \\
\hline Generic c vs Reference (48) & $111.87 \%(104.73-119.49)$ & $111.41 \%(104.41-118.90)$ & $106.58 \%(98.35-115.49)$ \\
\hline Generic a vs Generic b (48) & 102.64\% (96.09-109.63) & 102.74\% (96.27-109.64) & $103.26 \%(95.29-111.90)$ \\
\hline Generic b vs Generic c (48) & $92.18 \%$ (86.30-98.46) & $92.19 \%$ (86.39-98.38) & $96.74 \%(89.27-104.84)$ \\
\hline Generic a vs Generic c (48) & $94.61 \%(88.58-101.06)$ & $94.71 \%(88.75-101.08)$ & $99.89 \%(92.18-108.25)$ \\
\hline Cephalexin & MSR 0.008, CV 8.9\% & MSR 0.008, CV 8.9\% & MSR 0.040, CV 20.3\% \\
\hline Generic a vs Reference (32) & $99.46 \%(95.77-103.29)$ & $95.50 \%(92.98-99.16)$ & $107.53 \%(98.73-117.10)$ \\
\hline Generic b vs Reference (32) & $101.43 \%(97.67-105.34)$ & $101.18 \%(97.45-105.06)$ & $95.11 \%(87.33-103.58)$ \\
\hline Generic c vs Reference (32) & $98.65 \%$ (94.99-102.41) & $98.44 \%(94.81-102.21)$ & $105.52 \%(96.89-114.92)$ \\
\hline Generic a vs Generic b (32) & $94.39 \%$ (90.91-98.00) & $94.36 \%(90.88-97.97)$ & $113.06 \%(103.81-123.13)$ \\
\hline Generic b vs Generic c (32) & $102.79 \%(99.99-106.72)$ & 102.86\% (99.07-106.80) & $90.13 \%(82.76-98.16)$ \\
\hline Generic a vs Generic c (32) & $97.02 \%(93.44-100.73)$ & $97.06 \%(93.48-100.77)$ & 101.90\% (93.57-110.98) \\
\hline Ciprofloxacin & MSR 0.012, CV11.0\% & MSR 0.012, CV 11.0\% & MSR 0.020, CV14.2\% \\
\hline Generic a vs Reference (41) & $93.40 \%$ (89.67-97.29) & 92.99\% (89.28-96.86) & $94.20 \%(89.37-99.29)$ \\
\hline Generic b vs Reference (41) & $98.38 \%(94.45-102.47)$ & $97.51 \%(93.62-101.57)$ & $92.92 \%$ (88.15-97.94) \\
\hline Generic c vs Reference (41) & $101.77 \%$ (97.71-106.01) & 101.37\% (97.32-105.59) & 103.39\% (98.09-108.98) \\
\hline Generic a vs Generic b (41) & $94.94 \%$ (91.15-98.89) & $95.36 \%(91.55-99.33)$ & 101.38\% (96.18-106.86) \\
\hline Generic b vs Generic c (42) & $91.78 \%$ (88.11-95.60) & $91.74 \%$ (88.07-95.55) & $91.11 \%$ (86.44-96.04) \\
\hline Generic a vs Generic c (41) & $96.83 \%(93.01-100.81)$ & 96.39\% (92.59-100.35) & $90.16 \%(85.60-94.97)$ \\
\hline Clarithromycin & MSR 0.060, CV 24.9\% & MSR 0.057, CV 24.2\% & MSR 0.100, CV 32.4\% \\
\hline Generic a vs Reference (48) & $96.40 \%$ (88.64-104.85) & $96.91 \%(89.30-105.17)$ & $93.85 \%(84.22-104.60)$ \\
\hline Generic b vs Reference (47) & $102.52 \%(94.18-111.60)$ & $103.61 \%(95.39-112.54)$ & $96.28 \%(86.29-107.42)$ \\
\hline Generic c vs Reference (48) & $89.22 \%$ (82.04-97.04) & $89.83 \%$ (82.77-97.49) & 87.74\% (78.73-97.78) \\
\hline Generic a vs Generic b (47) & 93.97\% (86.32-102.29) & $93.42 \%(86.01-101.48)$ & $96.98 \%(86.92-108.21)$ \\
\hline Generic b vs Generic c (47) & $115.23 \%(105.85-125.43)$ & $115.70 \%(106.51-125.68)$ & 109.97\% (98.56-122.70) \\
\hline Generic a vs Generic c (48) & $108.05 \%$ (99.35-117.51) & $107.88 \%(88.41-117.08)$ & 106.97\% (95.98-119.21) \\
\hline
\end{tabular}


Table 2 Average bioequivalence among 3 randomly-selected generic products and reference product of 14 immediate-release, non-combinational, oral drugs (Continued)

\begin{tabular}{|c|c|c|c|}
\hline Drug & $A \cup C_{T}$ & $A \cup C_{1}$ & $C_{\max }$ \\
\hline Diclofenac & MSR 0.023, CV 15.3\% & MSR 0.022, CV 14.7\% & MSR 0.129, CV 37.1\% \\
\hline Generic a vs Reference (67) & $100.03 \%(95.74-104.52)$ & $100.19 \%(96.04-104.52)$ & $86.61 \%$ (78.08-96.08) \\
\hline Generic b vs Reference (68) & $99.80 \%$ (95.54-104.24) & 99.77\% (95.68-104.05) & $92.48 \%(83.43-102.51)$ \\
\hline Generic c vs Reference (68) & 103.74\% (99.32-108.36) & $104.01 \%(99.74-108.47)$ & $86.46 \%$ (78.00-95.83) \\
\hline Generic a vs Generic b (68) & $101.38 \%(97.06-105.89)$ & $101.50 \%(97.33-105.85)$ & $95.64 \%(86.28-106.01)$ \\
\hline Generic b vs Generic c (69) & $96.38 \%(92.30-100.64)$ & $96.06 \%(92.14-100.14)$ & 107.01\% (96.61-118.52) \\
\hline Generic a vs Generic c (68) & $96.71 \%(92.59-101.01)$ & $96.56 \%(92.59-100.70)$ & 100.79\% (90.93-111.72) \\
\hline Ibuprofen & MSR 0.012, CV 10.9\% & MSR 0.008, CV 9.1\% & MSR 0.026, CV 16.3\% \\
\hline Generic a vs Reference (27) & 106.31\%(101.06-111.83) & $104.65 \%(100.30-109.19)$ & 101.67\% (94.30-109.62) \\
\hline Generic b vs Reference (25) & $105.48 \%(100.05-111.20)$ & $102.94 \%(98.48-107.59)$ & $113.00 \%(104.47-122.23)$ \\
\hline Generic c vs Reference (26) & $106.30 \%(100.95-111.94)$ & $105.69 \%(101.21-110.36)$ & $89.11 \%$ (82.52-96.22) \\
\hline Generic a vs Generic b (26) & 102.27\% (97.11-107.69) & 103.13\% (98.76-107.69) & $92.17 \%$ (85.36-99.53) \\
\hline Generic b vs Generic c (26) & $98.38 \%(93.418-103.60)$ & $96.53 \%(92.44-100.80)$ & $126.47 \%(117.11-136.57)$ \\
\hline Generic a vs Generic c (27) & $99.87 \%$ (94.94-105.06) & 98.87\% (94.78-103.16) & $114.12 \%(105.85-123.04)$ \\
\hline Fluconazole & MSR 0.004, CV 6.3\% & MSR 0.004, CV 6.3\% & MSR 0.006, CV 7.8\% \\
\hline Generic a vs Reference (26) & $101.33 \%$ (98.34-104.42) & $102.23 \%(99.21-105.35)$ & 106.99\% (103.13-110.99) \\
\hline Generic b vs Reference (25) & $101.06 \%(98.01-104.21)$ & 101.39\% (98.33-104.55) & 109.79\% (105.75-113.99) \\
\hline Generic c vs Reference (25) & $105.66 \%(102.47-108.94)$ & $106.07 \%(102.86-109.36)$ & $109.00 \%(104.98-113.17)$ \\
\hline Generic a vs Generic b (25) & $100.38 \%(97.35-103.50)$ & 100.39\% (97.36-103.52) & $97.59 \%$ (93.99-101.32) \\
\hline Generic b vs Generic c (25) & $95.65 \%$ (92.77-98.63) & $96.21 \%(93.31-99.21)$ & 100.73\% (97.01-104.58) \\
\hline Generic a vs Generic c (25) & $96.01 \%$ (93.12-99.00) & $96.59 \%$ (93.68-99.60) & $98.30 \%(94.67-102.06)$ \\
\hline Metformin & MSR 0.019, CV 13.8\% & MSR 0.019, CV 13.8\% & MSR 0.027, CV 16.5\% \\
\hline Generic a vs Reference (48) & 93.19\% (88.89-97.69) & $92.44 \%$ (88.17-96.91) & $93.05 \%(87.96-98.44)$ \\
\hline Generic b vs Reference (48) & $97.70 \%$ (93.19-102.42) & $97.31 \%(92.82-102.01)$ & 98.45\% (93.06-104.15) \\
\hline Generic c vs Reference (49) & 96.06\% (91.68-100.66) & $95.51 \%(91.15-100.08)$ & $95.07 \%(89.92-100.52)$ \\
\hline Generic a vs Generic b (49) & $100.80 \%$ (97.76-103.94) & $95.06 \%(90.72-99.61)$ & 94.41\% (89.298-99.82) \\
\hline Generic b vs Generic c (49) & $95.60 \%$ (92.71-98.57) & $102.25 \%(97.61-107.17)$ & 104.15\% (98.50-110.11) \\
\hline Generic a vs Generic c (49) & $96.36 \%$ (93.46-99.36) & $97.23 \%$ (92.79-101.88) & $98.33 \%(93.00-103.96)$ \\
\hline Metronidazole & MSR 0.003, CV 5.5\% & MSR 0.003, CV 5.5\% & MSR 0.010, CV 10.0\% \\
\hline Generic a vs Reference (28) & $108.73 \%$ (106.05-111.48) & $108.96 \%(106.28-111.72)$ & 109.47\% (104.60-114.58) \\
\hline Generic b vs Reference (27) & $99.569 \%(97.07-102.14)$ & $99.82 \%(97.31-102.39)$ & $97.60 \%(93.17-102.24)$ \\
\hline Generic c vs Reference (28) & 97.439\% (95.04-99.90) & $97.46 \%$ (95.06-99.92) & $100.53 \%(96.05-105.22)$ \\
\hline Generic a vs Generic b (27) & $109.349 \%(106.60-112.17)$ & $109.32 \%(106.57-112.14)$ & $111.57 \%(106.50-116.88)$ \\
\hline Generic b vs Generic c (27) & $102.05 \%$ (99.49-104.68) & $102.29 \%(99.72-104.93)$ & 96.95\% (92.55-101.56) \\
\hline Generic a vs Generic c (28) & $111.59 \%(108.84-114.41)$ & $111.81 \%(109.05-114.63)$ & $108.90 \%(104.05-113.98)$ \\
\hline Omeprazole & MSR 0.035, CV 18.9\% & MSR 0.035, CV 18.9\% & MSR 0.066, CV 26.1\% \\
\hline Generic a vs Reference (74) & $97.49 \%(92.62-102.62)$ & 97.44\% (92.57-102.56) & $90.57 \%(84.41-97.17)$ \\
\hline Generic b vs Reference (73) & $96.21 \%(91.37-101.30)$ & $97.05 \%(92.17-102.19)$ & 84.85\% (79.04-91.08) \\
\hline Generic c vs Reference (74) & $98.14 \%(93.24-103.30)$ & 98.09\% (93.19-103.25) & $87.82 \%(81.85-94.23)$ \\
\hline Generic a vs Generic b (73) & 101.33\% (96.24-106.70) & $100.34 \%$ (95.30-105.66) & 106.59\% (99.30-114.4) \\
\hline Generic b vs Generic c (74) & $97.59 \%(92.71-102.72)$ & 98.55\% (93.63-103.73) & $96.35 \%$ (89.80-103.37) \\
\hline Generic a vs Generic c (74) & $99.34 \%$ (94.38-104.56) & 99.33\% (94.37-104.55) & 103.13\% (96.12-110.64) \\
\hline
\end{tabular}


Table 2 Average bioequivalence among 3 randomly-selected generic products and reference product of 14 immediate-release, non-combinational, oral drugs (Continued)

\begin{tabular}{llll}
\hline Drug & AUC & AUC & $C_{\max }$ \\
\hline Paracetamol & MSR 0.008, CV 8.8\% & MSR 0.008, CV 9.1\% & MSR 0.031, CV 17.6\% \\
Generic a vs Reference (40) & $91.57 \%(88.62-94.62)$ & $91.81 \%(88.76-94.96)$ & $104.99 \%(98.30-112.14)$ \\
Generic b vs Reference (38) & $99.69 \%(96.35-103.14)$ & $99.66 \%(96.22-103.22)$ & $103.48 \%(96.71-110.73 \%)$ \\
Generic c vs Reference (39) & $97.95 \%(94.71-101.29)$ & $97.86 \%(94.53-101.30)$ & $101.17 \%(94.63-108.15)$ \\
Generic a vs Generic b (38) & $100.01 \%(96.71-103.43)$ & $100.26 \%(96.85-103.79)$ & $101.76 \%(95.18-108.78)$ \\
Generic b vs Generic c (38) & $94.29 \%(91.18-97.52)$ & $94.29 \%(91.09-97.62)$ & $102.27 \%(95.57-109.43)$ \\
Generic a vs Generic c (39) & $102.11 \%(98.74-105.60)$ & $102.46 \%(98.97-106.07)$ & $104.11 \%(97.38-111.30)$ \\
Ranitidine & MSR 0.021, CV 14.6\% & MSR 0.020, CV 14.2\% & MSR 0.047, CV 21.9\% \\
Generic a vs Reference (70) & $102.68 \%(98.57-106.96)$ & $102.43 \%(98.43-106.59)$ & $105.26 \%(99.02-111.89)$ \\
Generic b vs Reference (71) & $102.54 \%(98.47-106.79)$ & $102.50 \%(98.52-106.64)$ & $98.21 \%(92.43-104.34)$ \\
Generic c vs Reference (72) & $101.84 \%(97.82-106.02)$ & $101.81 \%(97.89-105.89)$ & $104.51 \%(98.40-111.00)$ \\
Generic a vs Generic b (70) & $100.30 \%(96.38-104.37)$ & $100.29 \%(96.37-104.37)$ & $107.89 \%(101.49-114.69)$ \\
Generic b vs Generic c (71) & $100.27 \%(96.38-104.31)$ & $100.34 \%(96.45-104.39)$ & $93.64 \%(88.13-99.49)$ \\
Generic a vs Generic c (70) & $100.30 \%(96.38-104.38)$ & $100.38 \%(96.45-104.46)$ & $100.66 \%(94.70-107.01)$ \\
\hline
\end{tabular}

$\mathrm{AUC}_{\mathrm{T}}$ is the area-under-the-concentration-time curve to last measured concentration. $A U C_{1}$ is $A U C$ extrapolated to infinity. $C_{\text {max }}$ is maximum concentration. Data represent geometric mean ratios and unadjusted $90 \%$ confidence intervals. The number of subjects analyzed in each comparison is presented between parentheses in the first column. MSR is mean square residual from analysis of variance (ANOVA). CV is intra-subject coefficient of variation calculated as $100 \times$ (exp(MSR)- 1$)^{0.5}$. Confidence intervals that cross the $80.00 \%-125.00 \%$ bioequivalence limits are bolded

deviations were $>13$ percentage points and $81.0 \%, 81.0 \%$, and $59.5 \%$, respectively, were $<6$ percentage points.

Figure 6 (a) depicts $\mathrm{BE}$ analysis of $\mathrm{AUC}_{\mathrm{Reftmax}}$ among the three generic products of each of the 14 drugs. The data are also summarized in Additional file 8. Twenty three $(54.8 \%)$ of the $90 \%$ CIs failed to show bioequivalence. In addition, 5 (11.9\%) showed bioinequivalence. Figure 6 (b) depicts $\mathrm{BE}$ analysis of $\mathrm{AUC}_{72}$ among the three generic products of the two drugs with long halflife. BE was demonstrated by all of the six $90 \%$ CIs. The data are also summarized in Additional file 9.

\section{Individual pharmacokinetic parameter ratios among 3 on-} market generic products of $\mathbf{1 4}$ drugs

There were 1952 individual generic-generic comparisons. The percentages of individual $\mathrm{AUC}_{\mathrm{T}}, \mathrm{AUC}_{\mathrm{I}}$, and $\mathrm{C}_{\mathrm{max}}$, ratios that were outside the $\pm 25 \%$ range are presented in Fig. 7. On average, $17 \%$ of the $\mathrm{AUC}_{\mathrm{T}}$ ratios (ranging from $1 \%$ for metronidazole and fluconazole to $40 \%$ for clarithromycin), $16 \%$ of the $\mathrm{AUC}_{\mathrm{I}}$ ratios (ranging from $1 \%$ for metronidazole and fluconazole to $38 \%$ for clarithromycin), and $32 \%$ of the $\mathrm{C}_{\max }$ ratios (ranging from $5 \%$ for fluconazole to $59 \%$ for diclofenac) were outside the $\pm 25 \%$ range. Further, individual $\mathrm{AUC}_{\mathrm{T}}, \mathrm{AUC}_{\mathrm{I}}$, and $\mathrm{C}_{\max }$ ratios were within the $\pm 25 \%$ range in $>75 \%$ of individuals for $71 \%, 71 \%$, and $29 \%$ of the 14 drugs, respectively,

Out of 161 and $76 \mathrm{AUC}_{72}$ individual ratios for amlodipine and fluconazole, $19 \%$ and 1\%, respectively, were outside the $\pm 25 \%$ range (compared to $25 \%$ and $1 \%$, respectively, for $\mathrm{AUC}_{\mathrm{T}}$ ).
Figure 8 depicts the percentages of individual generic/ generic $\mathrm{T}_{\max }$ and $A U C_{\text {Reftmax }}$ ratios that were outside the $\pm 25 \%$ range. On average, $58 \%$ of the $\mathrm{T}_{\max }$ ratios (ranging from $42 \%$ for amlodipine to $73 \%$ for fluconazole) and $52 \%$ of the $\mathrm{AUC}_{\text {Reftmax }}$ ratios (ranging from $18 \%$ for fluconazole to $82 \%$ for omeprazole) were outside the $\pm 25 \%$ range. Individual $\mathrm{T}_{\max }$ and $\mathrm{AUC}_{\text {Reftmax }}$ ratios were within the $\pm 25 \%$ range in $>75 \%$ of individuals for $0 \%$ and $7 \%$ of the 14 drugs, respectively.

\section{Discussion}

We assessed the adequacy of the commonly-used $\mathrm{BE}$ standards and of their application in a developing country through determining $\mathrm{BE}$ extent between onmarket generic and reference drug products and among reference-bioequivalent generic drug products. We studied 42 generic products of 14 immediate-release, non-combinational, oral drugs with the highest number of generic products on the Saudi market. We conducted a four-product, four-period, four-sequence, sequencerandomized, crossover $\mathrm{BE}$ study with a planned power of 0.9 on a reference and three randomly-selected generic products of each of the 14 drugs. For each drug, we computed six pairwise $90 \%$ CIs on geometric mean ratios of $\mathrm{AUC}_{\mathrm{T}}, \mathrm{AUC}_{\mathrm{I}}, \mathrm{C}_{\max }, \mathrm{AUC}_{\text {Reftmax, }}$ and $\mathrm{AUC}_{72}$ without and with adjustment for multiple comparisons and determined percentages of individual untransformed ratios that fell outside the $\pm 25 \%$. We found that: 1) Onmarket generic drug products continue to be referencebioequivalent. 2) Reference-bioequivalent generic products are bioequivalent to each other. 3) Reference-generic 


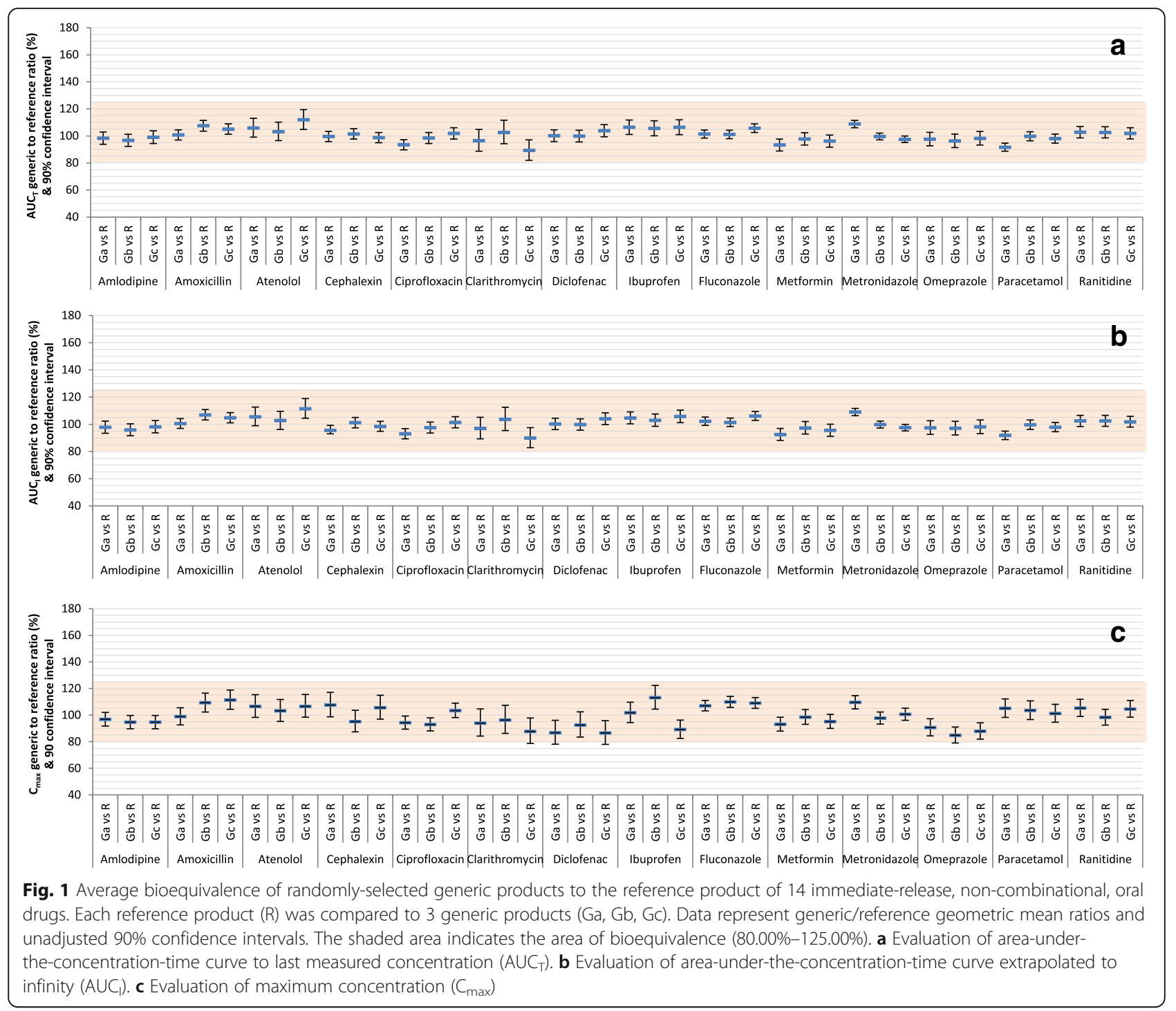

and generic-generic average deviations are small and similar. 4) Reference-generic and generic-generic $C_{\max }$ intra-subject variations are large but similar. 5) Two thirds of generic-reference and generic-generic $\mathrm{AUC}_{\text {Reftmax }}$ comparisons failed to show average bioequivalence/ showed bioinequivalence.

The number of generic products for an off-patent drug is usually related to its market size. Therefore, it is reasonable to assume that the 14 drugs that we studied are among the commonly prescribed drugs in Saudi Arabia. They happened to include drugs for which rapid onset of action is clinically relevant (paracetamol, ibuprofen, diclofenac), drugs that are used in chronically and for which the concept of switchability is relevant (metformin, amlodipine), drugs with long half-life (fluconazole, amlodipine), and highly variable drugs (clarithromycin, diclofenac), but not NTI drugs. Almost all of the generic products were manufactured in Saudi Arabia or in a Middle Eastern state.

\section{Marketed generic products of immediate-release, non- computational, oral drugs continue to be bioequivalent to their corresponding reference products}

A generic drug product is commonly approved for continued marketing based on a single pre-marketing study demonstrating $\mathrm{BE}$ to its reference product; retesting of BE post-marketing is not routinely required. Our results confirm the validity of such practice. Using the $80.00-125.00 \%$ BE range, we found that $100 \%$ of the $\mathrm{AUC}_{\mathrm{T}}$ and $\mathrm{AUC}_{\mathrm{I}}$ generic-reference $90 \%$ CIs showed $\mathrm{BE}$ and only $9.5 \%$ of the $C_{\max } 90 \%$ CIs barely failed to show BE. Even after adjusting for 6 comparisons, only $2.4 \%$ of the $\mathrm{AUC}_{\mathrm{T}}$ and $\mathrm{AUC}_{\mathrm{I}} 90 \% \mathrm{CIs}$ and $21.4 \%$ of the $\mathrm{C}_{\max }$ 90\% CIs failed to show BE. Our results are in line with some $[17,22]$ but not all $[15,16]$ published studies. 

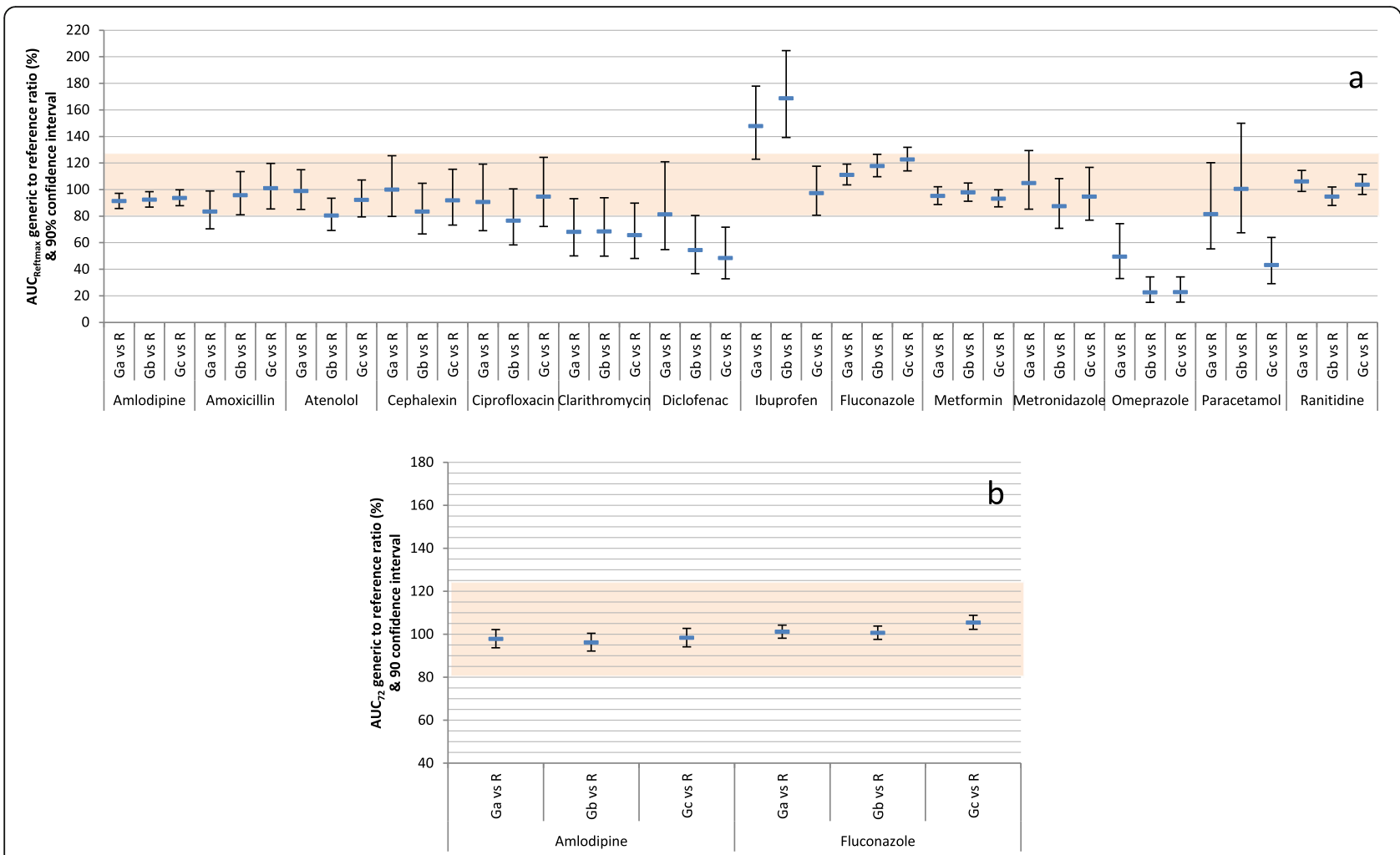

Fig. 2 Average bioequivalence of randomly-selected generic products to the reference product of 14 immediate-release, non-combinational, oral drugs. Each reference product (R) was compared to 3 generic products (Ga, Gb, Gc). Data represent generic/reference geometric mean ratios and unadjusted $90 \%$ confidence intervals. The shaded area indicates the area of bioequivalence (80.00\%-125.00\%). a Evaluation of area-underthe-concentration-time curve to time of maximum concentration of reference product, calculated for each subject (AUC $C_{\text {Reftmax }}$ ). $\mathbf{b}$ Evaluation of areaunder-the-concentration-time curve truncated to $72 \mathrm{~h}\left(A \cup C_{72}\right)$. Only 2 drugs (amlodipine and fluconazole) in this study have terminal half-life $>72 \mathrm{~h}$

Previous studies evaluated generic products on other national markets, examined only one [17] or two [16, $22]$ generic products of a single drug, or were not performed in vivo [15].

The outcome of a crossover BE study is affected by its sample size and intra-subject variability [57]. We estimated intra-subject CVs from published studies and planned each of the 14 studies to have a power of 0.9 . It is of note that for the 4 drugs that failed to show $\mathrm{BE}$ in some of the comparisons (clarithromycin, diclofenac, ibuprofen, and omeprazole), current study intra-subject $\mathrm{CVs}$ were larger than estimated (Additional file 2). Intra-subject variability can be related to inter-product variability; however, it can be also attributed to the drug substance itself (being readily affected by intra-subject physiological variability), intraproduct variability, analytical variability, or unexplained random variability [57]. In fact, in a separate study [58] that compared the reference ibuprofen product used in this study to itself, using the same settings and a larger sample size, the $\mathrm{C}_{\max } 90 \% \mathrm{CI}$ also failed to show $\mathrm{BE}$. This suggests that at least some of the failures to show $\mathrm{BE}$ in the current study may not be due to real genericreference (inter-product) differences.
We found that the mean deviation of the generic/reference ratio from $100 \%$ was $3.2 \%, 3.2 \%$, and $5.4 \%$ for $\mathrm{AUC}_{\mathrm{T}}$, $\mathrm{AUC}_{\mathrm{I}}$, and $\mathrm{C}_{\max }$, respectively, and that the deviation was $<10$ percentage points in $95.2 \%, 95.2 \%$, and $81.0 \%$ of the 42 comparisons. Similarly, the US FDA found a mean deviation of $3.47 \%$ for $\mathrm{AUC}_{\mathrm{T}}$ and $4.29 \%$ for $\mathrm{C}_{\max }$ in one retrospective study [59] and $3.56 \%$ for $\mathrm{AUC}_{\mathrm{T}}$ and $4.35 \%$ for $\mathrm{C}_{\max }$ in another [60], and that in about $98 \%$ of the studies, the $\mathrm{AUC}_{\mathrm{T}}$ difference was $<10 \%$ [60]. Further, a reanalysis of 141 US FDA-approved antiepileptic generic products found that generic and reference $\mathrm{AUC}_{\mathrm{T}}$ and $\mathrm{C}_{\max }$ differed by $<15 \%$ in $99 \%$ and $89 \%$ of BE studies, respectively [28]. Consistent with these BE findings, several metaanalysis and reviews showed that there is no evidence that cardiovascular [18, 19], antiepileptic [20], or immunosuppressive [21] reference drug products are superior to their generic counterparts in terms of efficacy or side effects.

Reference-bioequivalent generic drug products continue to be underused world-wide, mainly due to mistrust by healthcare professionals [2] and patients [3], in a way that may be dependent on maturity of the country's healthcare system $[2,5,6]$. The misbelief that generic medicines are counterfeits and the placebo effect of packaging and price 


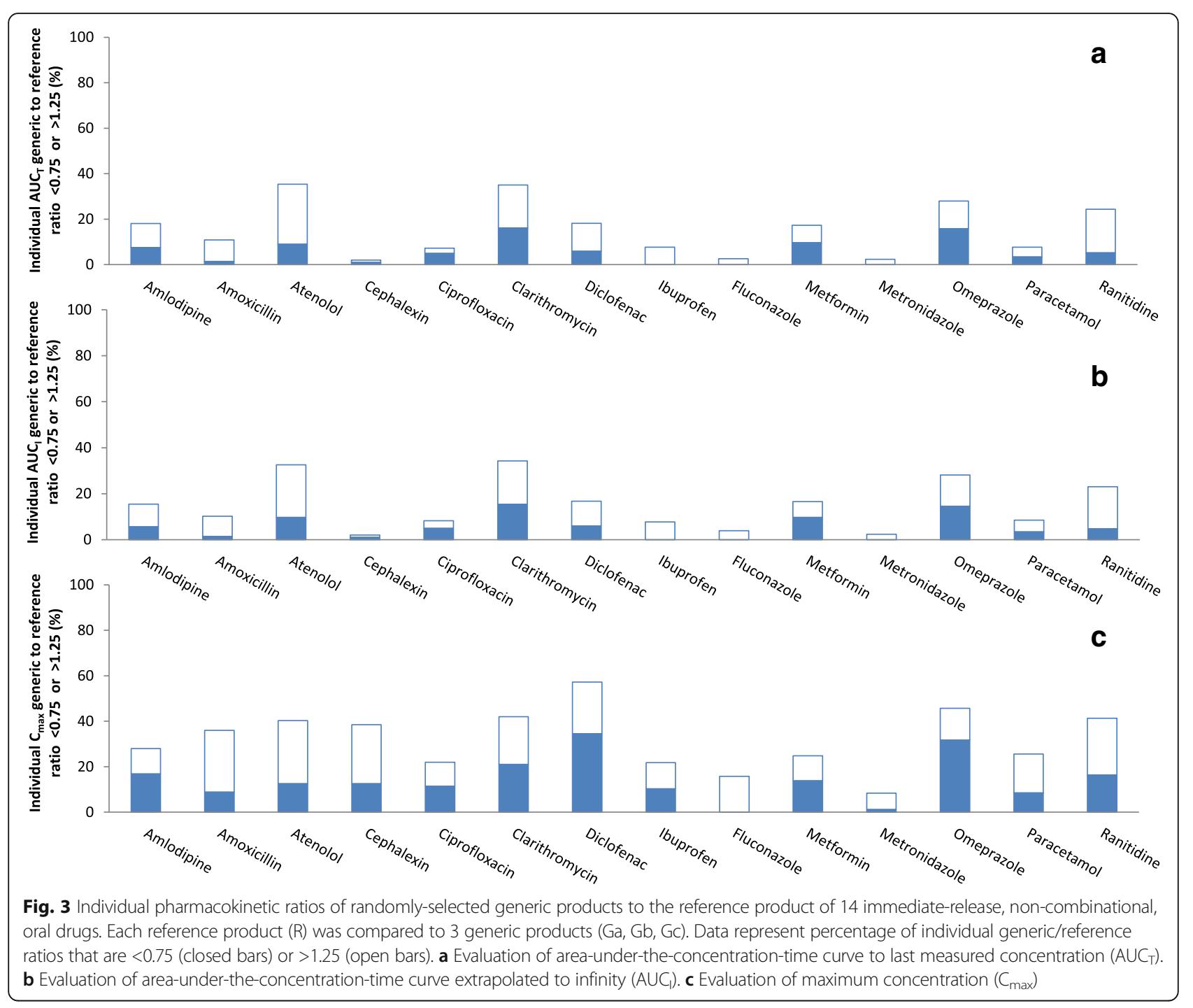

differential are important to consider [61]. Further, prescribing a generic product by its brand name rather than its non-proprietary name (generic prescribing) may better convey the impression of individuality and improve patients' acceptance [62, 63]. Importantly, information availability to healthcare professionals and patients has been identified as a facilitator of generic products uptake $[4,39]$. Our results provide strong supporting evidence of the post-marketing quality of generic products and of the adequacy of the current BE standards.

\section{Marketed, reference-bioequivalent, generic products of immediate-release, non-combinational, oral drugs are bioequivalent to each other}

Commonly, there are several same-market drug products that are linked by a chain of reference; theoretical concerns have been raised that reference-bioequivalent generic products may not be bioequivalent to each other if their $\mathrm{BE}$ point estimates were on the opposite sides within the $\mathrm{BE}$ range [23, 24]. Simulation studies predicted that two reference-bioequivalent generic products are likely to be equivalent to each other only under relatively restricted conditions [29, 30]. However, using reference-normalized data to indirectly estimate $90 \%$ CIs, analysis of $19 \mathrm{BE}$ studies on 2 anti-epileptic drugs showed generic-generic $\mathrm{BE}$ in almost all cases [26] and analysis of $120 \mathrm{BE}$ studies on three immunosuppressants as well as six selected drugs showed $\mathrm{BE}$ in $90 \%$ of $\mathrm{AUC}_{\mathrm{T}}$ and $87 \%$ of $\mathrm{C}_{\max }$ comparisons with mean absolute deviation from $100 \%$ of $4.5 \%$ for $\mathrm{AUC}_{\mathrm{T}}$ and $5.1 \%$ for $C_{\max }$ [27]. Further, a similar analysis of US FDA-approved antiepileptic generic products found that $\mathrm{AUC}_{\mathrm{T}}$ and $\mathrm{C}_{\max }$ differed by $>15 \%$ in $17 \%$ and $39 \%$ of simulated generic-generic switches, respectively [28]. Nevertheless, there is little direct empirical evidence regarding the extent of $\mathrm{BE}$ among reference-bioequivalent generic products; two amoxicillin generic products did not show $\mathrm{BE}$ [16], whereas two metformin generic products [22] and the two most disparate generic lamotrigine products [31] did. 

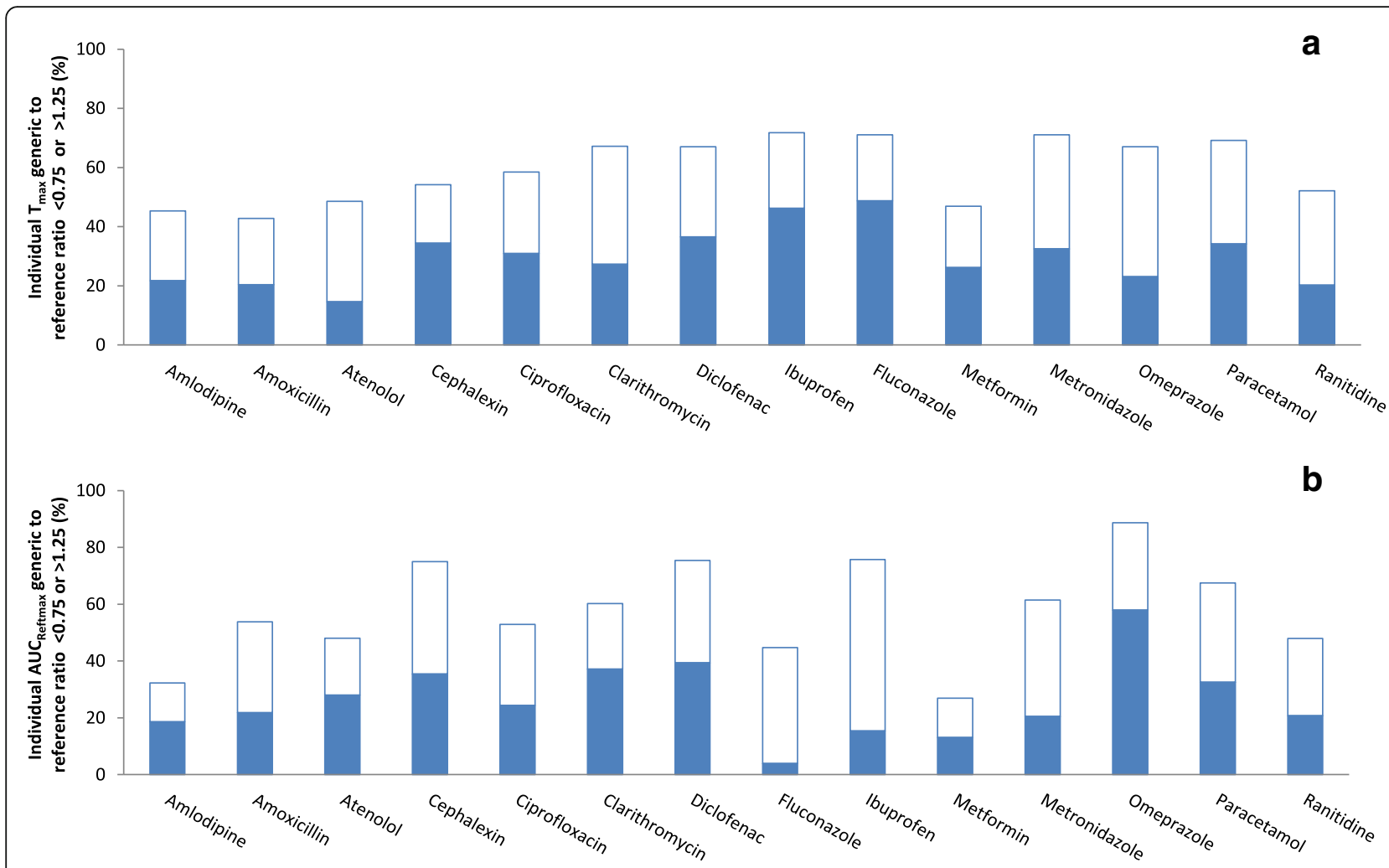

Fig. 4 Individual pharmacokinetic ratios of randomly-selected generic products to the reference product of 14 immediate-release, noncombinational, oral drugs. Each reference product (R) was compared to 3 generic products (Ga, Gb, Gc). Data represent percentage of individual generic/reference ratios that are $<0.75$ (closed bars) or $>1.25$ (open bars). a Evaluation of time of maximum concentration ( $\left.T_{\text {max }}\right)$. $\mathbf{b}$ Evaluation of area-under-the-concentration-time curve to time of maximum concentration of reference product, calculated for each subject (AUC $C_{\text {Reftmax }}$ )

In our prospective study of 42 direct generic-generic $\mathrm{BE}$ comparisons, only one (2.4\%) comparison failed to show $\mathrm{BE}$ because of $\mathrm{C}_{\max }$ and one because of $\mathrm{AUC}_{\mathrm{T}}$ and $\mathrm{AUC}_{\mathrm{I}}$. After adjusting for 6 comparisons, the percentages were $2.4 \%$ and $14.3 \%$, respectively. Further, mean deviation of generic/generic ratio from $100 \%$ was only $2.5 \%, 2.6 \%$, and $3.3 \%$ for $\mathrm{AUC}_{\mathrm{T}}, \mathrm{AUC}_{\mathrm{I}}$, and $\mathrm{C}_{\max }$, respectively, and the deviation was $<10$ percentage points in $95.2 \%, 95.2 \%$, and $88.1 \%$ of the 42 comparisons. Our results provide strong empirical evidence that it is very unlikely for two reference-bioequivalent generic products not to be bioequivalent to each other. Interestingly, in our study, mean deviation of generic/reference ratios from $100 \%$ was in the 6-13 percentage points range in $21.4 \%, 19 \%$, and $40.5 \%$ of the $\mathrm{AUC}_{\mathrm{T}}$ and, $\mathrm{AUC}_{\mathrm{I}}$, and $\mathrm{C}_{\max }$ comparisons, respectively. This suggests that, contrary to the result of previous simulation study [29], even when the bioavailability difference between generic and reference products is in the 6-13 percentage points range, reference-bioequivalent generic products are still likely to be bioequivalent.

Theoretically, the change in drug exposure resulting from generic-generic substitution might be expected to be more pronounced than the change resulting from generic-reference substitution [23, 24]. However, our results indicate that the two changes in exposure are similar. Mean absolute deviation of point estimates in percentage points was 3.2 vs. 2.5 for $\mathrm{AUC}_{\mathrm{T}}, 3.2$ vs. 2.6 for $\mathrm{AUC}_{\mathrm{I}}$, and 5.4 vs. 3.3 for $\mathrm{C}_{\max }$ in the genericreference and generic-generic comparisons, respectively. Further, the deviations were $<10$ percentage points in similar proportions of the two types of comparisons.

\section{Generic-reference and generic-generic intra-subject variability of bioequivalent drug products}

Since average BE focuses on mean difference rather than difference between variances or subject-by-product interaction, it is possible that a patient on a referencebioequivalent but low-quality generic product may be sometimes overdosed and sometimes underdosed and that a patient using two bioequivalent products may have the highest drug exposure with one product and the lowest with another [64]. Such possibilities may be of particular concern when switching patients form one NTI drug product to another [24] and are usually reflected in individual ratios of the pharmacokinetic parameters. Few published studies have addressed $\mathrm{BE}$ at the individual level $[17,24,25]$. Despite having $90 \%$ CIs within the $80-125 \%$ 


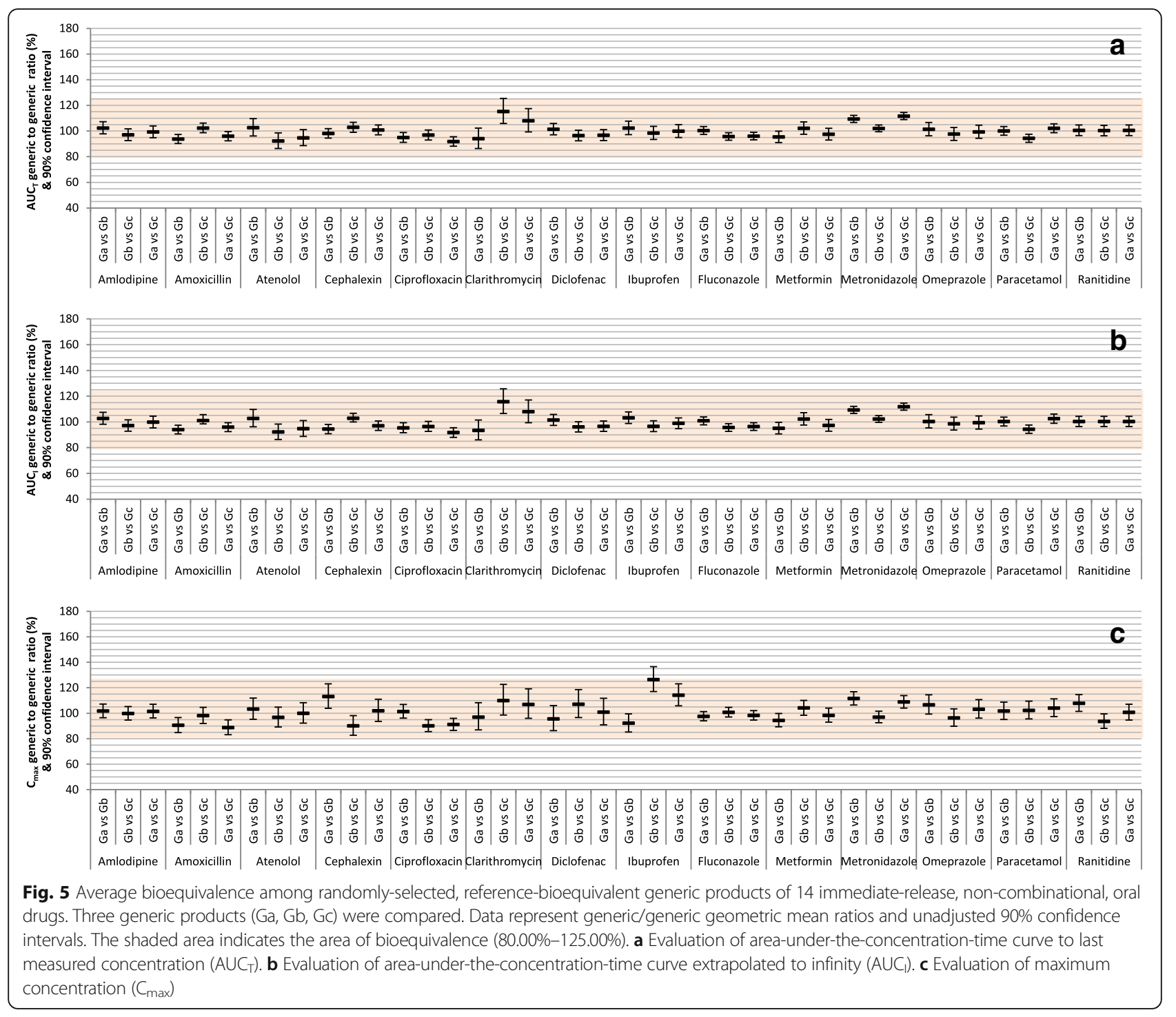

limits, $18 \%$ and $38 \%$ of individual cyclosporine generic/reference AUC and $\mathrm{C}_{\max }$ ratios, respectively, were $<0.80$ [24] and $0 \%$ of individual lamotrigine generic/reference AUC and $\mathrm{C}_{\max }$ ratios and $3 \%$ and $18 \%$ of same-product, generic/generic $\mathrm{AUC}$ and $\mathrm{C}_{\max }$ ratios, respectively, were outside the $\pm 25 \%$ range [17]. A simulation study (assuming 20\% inter-subject variability and 10\% intra-subject variability) predicted that when mean generic product's AUC is $80 \%$ to $123.5 \%$ of reference product's AUC, $3-4.6 \%$ and $9-12 \%$ of individual generic/reference and generic/generic AUC ratios, respectively, would fall outside the $0.67-1.5$ range [25].

We found that $16 \%$ and $17 \%$ of individual generic/reference and generic/generic ratios, respectively, were outside the $\pm 25 \%$ range in for $\mathrm{AUC}_{\mathrm{T}}, 15 \%$ and $16 \%$ for $\mathrm{AUC}_{\mathrm{T}}$, and $32 \%$ and $32 \%$ for $C_{\max }$. Further, individual generic/reference and generic/generic $\mathrm{AUC}_{\mathrm{T}}, \mathrm{AUC}_{\mathrm{I}}$, and $\mathrm{C}_{\max }$ ratios fulfilled the $75 / 75$ rule for $79 \%$ and $71 \%, 79 \%$ and $71 \%$, and $36 \%$ and $29 \%$ of the 14 drugs, respectively. Based on a relatively large number of drug products, our results document the extent of intra-subject variability that would be expected despite fulfilment of average BE criteria and strongly suggest that the extents of generic-generic switchability and generic-reference switchability are similar.

It is not clear how much of the observed intra-subject variability is due to inter-product rather than intra-product variability. In the simulation study, $11.1 \%$ of the reference/ reference AUC ratios were predicted to fall outside the 0.8-1.25 range [25]. Further, $3 \%$ and $9 \%$ of individual lamotrigine reference/reference AUC and $\mathrm{C}_{\max }$ ratios [17] and $23 \%, 30 \%$, and $30 \%$ of individual caffeine $\mathrm{AUC}_{\mathrm{T}}, \mathrm{AUC}_{\mathrm{I}}$, and $C_{\max }$ ratios [65], respectively, were outside the $\pm 25 \%$ range. Furthermore, when the cephalexin, ibuprofen, and paracetamol reference products used in this study were compared to themselves; respectively, $2 \%, 17 \%$, and $2 \%$ of the individual ratios were outside the $\pm 25 \%$ range for $\mathrm{AUC}_{\mathrm{T}}$ 


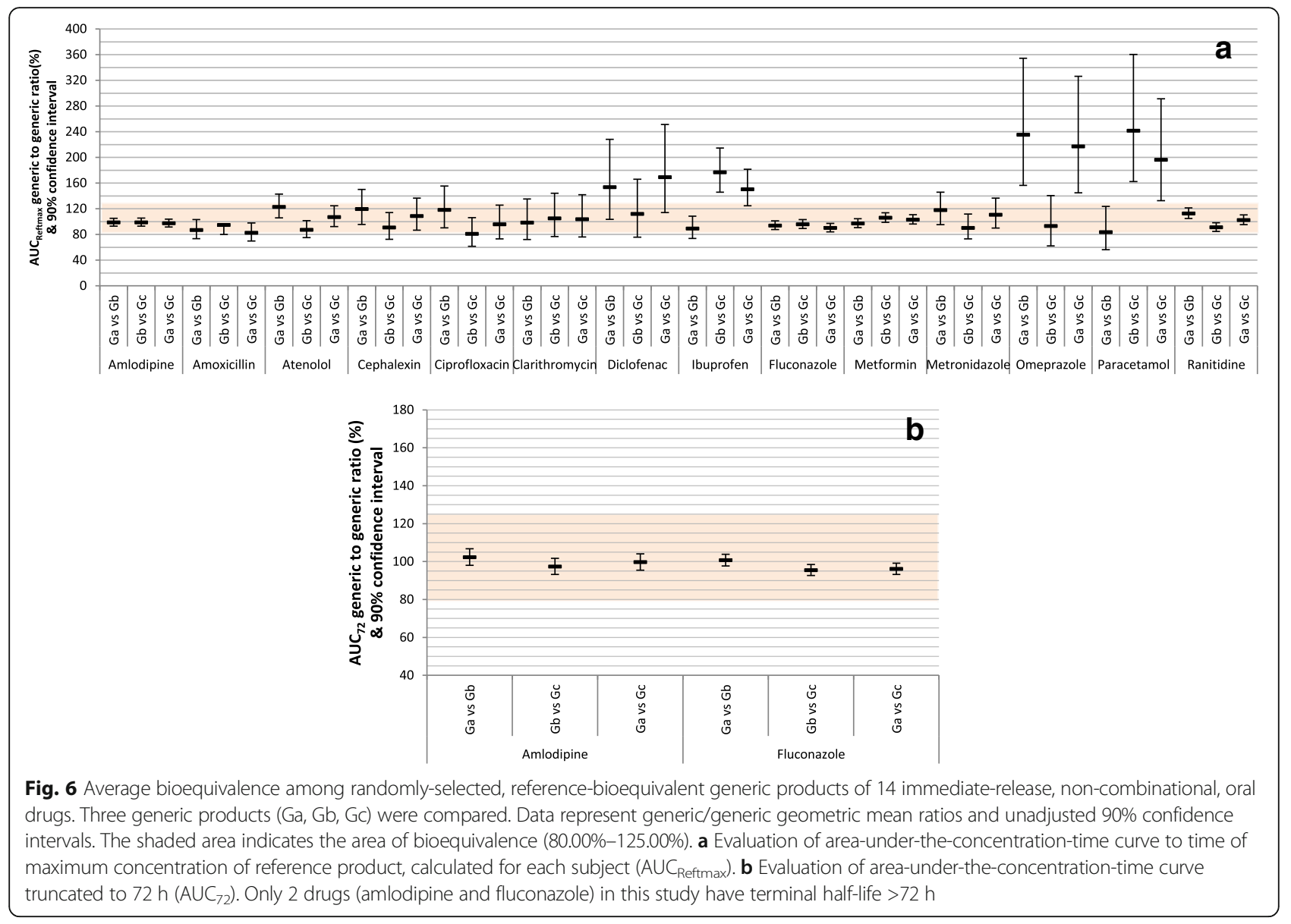

(compared to $2 \%, 8 \%$, and $8 \%$ of the generic-reference ratios in the current study), $4 \%, 3 \%$, and $2 \%$ for $\mathrm{AUC}_{\mathrm{I}}$, (compared to $2 \%, 8 \%$, and $9 \%$ of the generic-reference ratios in the current study), and $25 \%, 33 \%$, and $45 \%$ for $\mathrm{C}_{\max }$, (compared to $39 \%, 22 \%$, and $26 \%$ of the genericreference ratios in the current study) [58]. Together, the data strongly indicate that a major part of the intra-subject variability seen in average BE studies may not be related to comparing two products but rather to factors such as study setting, drug assay, and random variations in subject's physiologic status (for example, gastric emptying, intestinal transit speed, and luminal $\mathrm{pH}$ ).

\section{Large variability in $A U C_{\text {Reftmax }}$ and $T_{\max }$ despite average bioequivalence}

When time of onset of drug effect is important because of therapeutic or toxic issues, it is recommended to perform non-parametric analysis of non-transformed $\mathrm{T}_{\max }$ values and/or evaluate the $90 \%$ CI of AUC truncated at reference $\mathrm{T}_{\max }$ median or at reference $\mathrm{T}_{\max }$, calculated for each subject $\left(\mathrm{AUC}_{\text {Reftmax }}\right)[7,8]$. Onset of effect may important for only few drugs in the current study, however, we used the data on all the 14 drugs to examine the behaviour of $\mathrm{T}_{\max }$ and $\mathrm{AUC}_{\mathrm{Reftmax}}$ in general.
We found that two thirds of generic-reference and generic-generic $\mathrm{AUC}_{\text {Reftmax }}$ comparisons failed to show BE or showed bioinequivalence. Further, on average, $60 \%$ and $58 \%$ of generic/reference and $58 \%$ and $52 \%$ of generic/generic individual $\mathrm{T}_{\max }$ and $\mathrm{AUC}_{\text {Reftmax }}$ ratios, respectively, were outside the $\pm 25 \%$ range. Moreover, generic/reference and generic/generic individual $\mathrm{T}_{\max }$ and $\mathrm{AUC}_{\text {Reftmax }}$ ratios fulfilled the $75 / 75$ rule in only $0-7 \%$ of the 14 drugs. The results confirm that average $\mathrm{BE}$ testing using $\mathrm{AUC}_{\mathrm{T}}, \mathrm{AUC}_{\mathrm{I}}$, and $\mathrm{C}_{\text {max }}$ is insensitive to variability in $\mathrm{T}_{\max }$ and $\mathrm{AUC}_{\text {Reftmax }}$ and suggest that intra-subject variabilities of the two parameters are similar and do not depend on whether a generic product is compared to a reference product or to another generic product.

Some patients' bad impression of generic products may be theoretically related to their different onset of effect as compared to reference products. However, this is not likely because onset of effect is mostly related to pharmacodynamic rather than pharmacokinetic characteristics. Further, since $T_{\max }$ values are based on $C_{\max }$, which is, in turn, based on a single measurement of drug concentration, $\mathrm{T}_{\max }$ values are also very sensitive to study setting, subject's physiological status, assay variability, and random error. In 


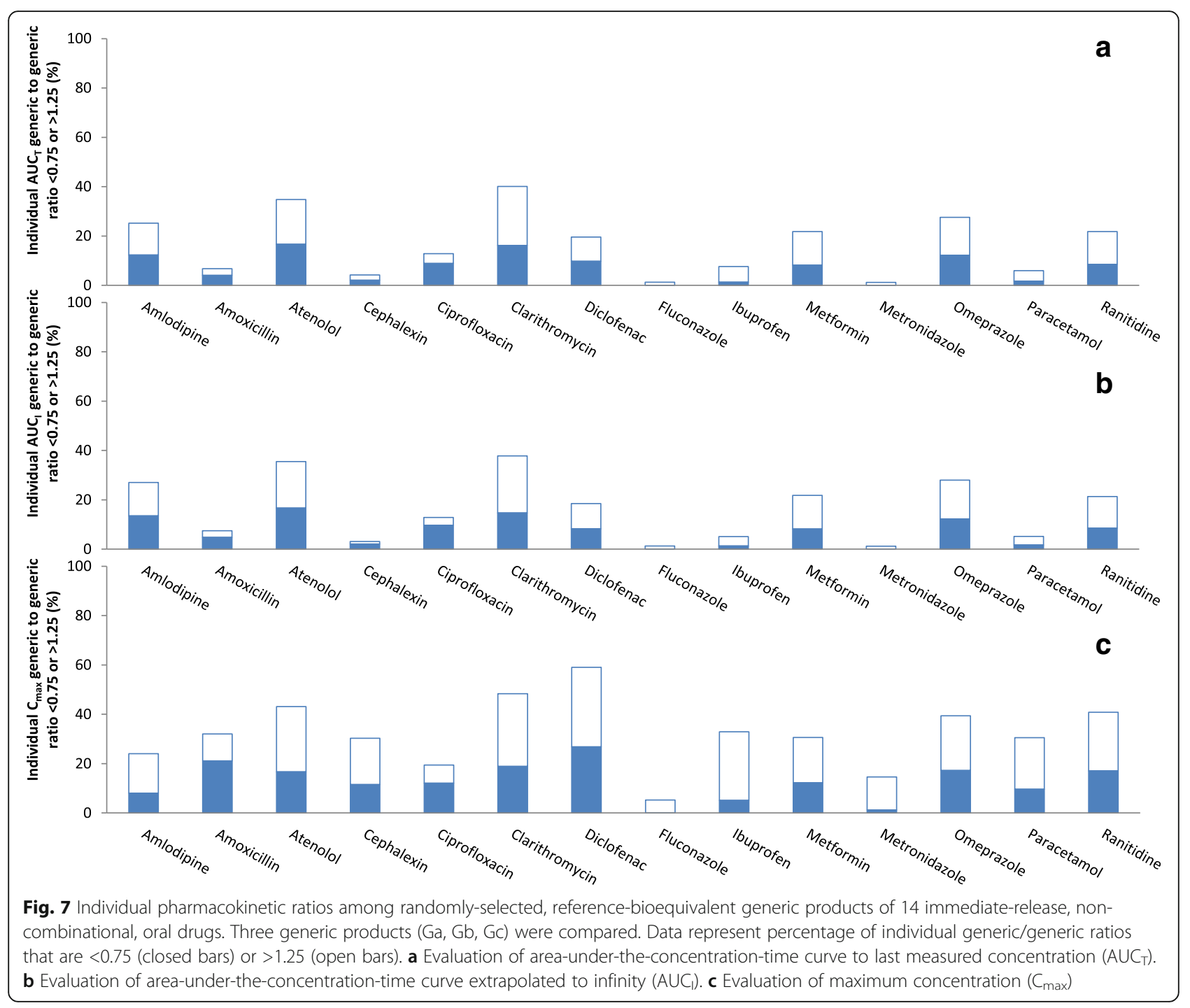

fact, when the cephalexin, ibuprofen, and paracetamol reference products used in this study were compared to themselves [58]; respectively, $46 \%, 63 \%$ and $71 \%$ of individual ratios were outside the $\pm 25 \%$ range for $\mathrm{T}_{\max }$ (compared to $54 \%, 72 \%$ and $69 \%$ of the generic-reference ratios in the current study) and $71 \%, 77 \%$ and $67 \%$ for AUC Reftmax $_{\text {(com- }}$ pared to $75 \%, 76 \%$ and $68 \%$ of the generic-reference ratios in the current study). This strongly indicates that most of the observed generic-reference and generic-generic intrasubject variability in $T_{\max }$ and $A U C_{\text {Reftmax }}$ is not due to inter-product differences and that the usefulness of $\mathrm{T}_{\max }$ and $\mathrm{AUC}_{\mathrm{Reftmax}}$ in $\mathrm{BE}$ evaluation may be very limited.

\section{$\mathrm{AUC}_{72}$ is as informative as $A U C_{T}$}

Two drugs in this study have long plasma half-life (around 49 and $29 \mathrm{~h}$ ); the half-life for the other 12 drugs was $<10 \mathrm{~h}$. We were able to demonstrate average $\mathrm{BE}$ in all genericreference and all generic-generic $\mathrm{AUC}_{\mathrm{T}}$ and $\mathrm{AUC}_{72}$ comparisons. Further, similar percentages of generic/reference and generic/generic individual $\mathrm{AUC}_{\mathrm{T}}$ and $\mathrm{AUC}_{72}$ ratios were outside the $\pm 25 \%$ range. The results lend further support to using $\mathrm{AUC}_{72}$ instead of $\mathrm{AUC}_{\mathrm{T}}$ for drugs with long plasma half-life [7-9].

\section{Limitations}

The interpretation of the results of this study may be limited by the following. 1) We only studied non-combinational drug products. However, BE standards for combinational and non-combinational products are the same and it can be assumed that the results apply to combinational products. 2) We only studied solid immediate-release drug products, thus our results may not apply to liquid or modified-release products. 3) Our results may not be generalizable to other solid immediate-release drugs on the Saudi market since the drugs we studied were not randomly selected. Short of more relevant statistics, the number of on-market generic products is a reasonable reflection of the extent of drug utilization. Further, the generic products in our study were 

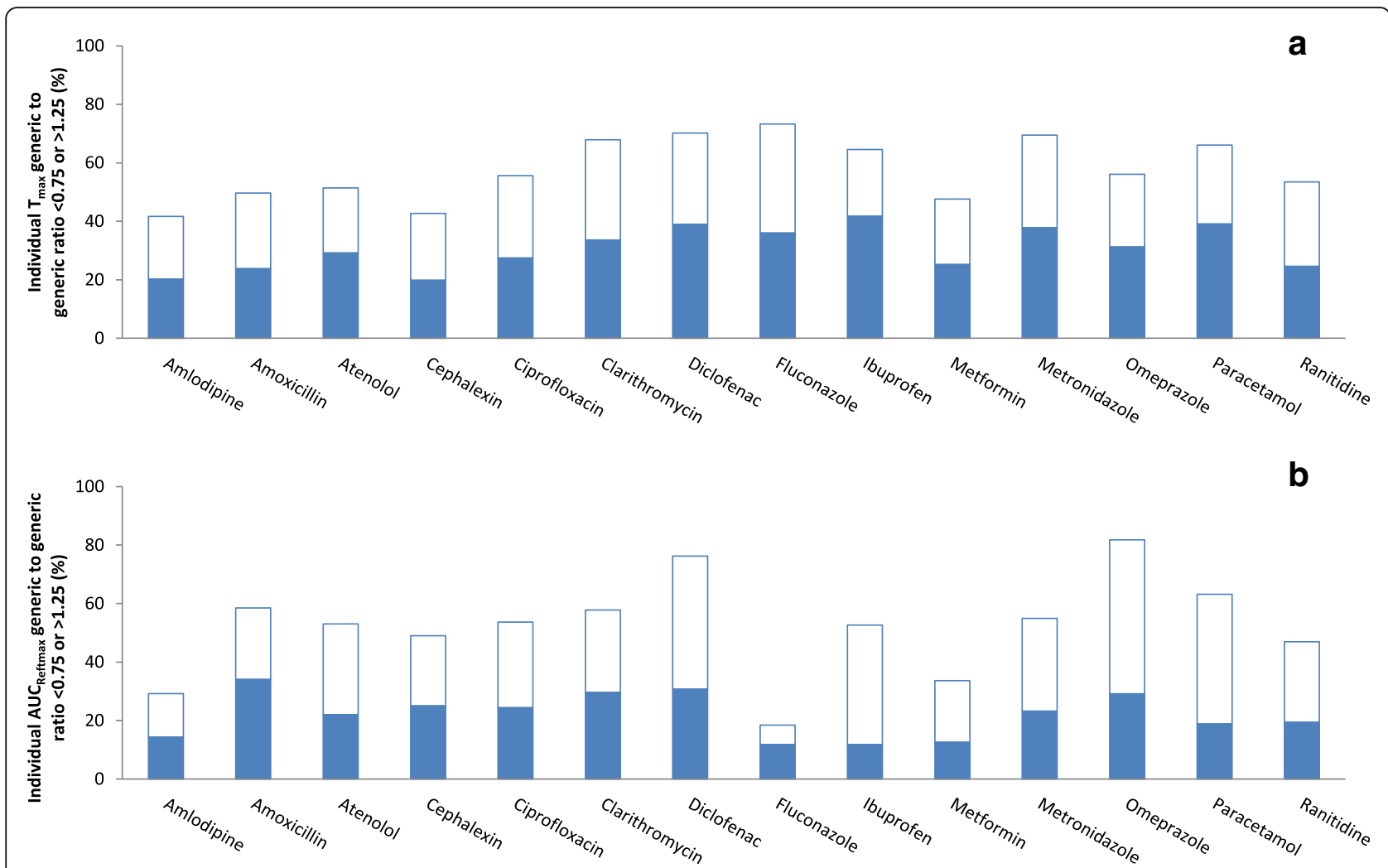

Fig. 8 Individual pharmacokinetic ratios among randomly-selected, reference-bioequivalent generic products of 14 immediate-release, noncombinational, oral drugs. Three generic products (Ga, Gb, Gc) were compared. Data represent percentage of individual generic/generic ratios that are $<0.75$ (closed bars) or $>1.25$ (open bars). a Evaluation of time of maximum concentration $\left(T_{\max }\right)$. $\mathbf{b}$ Evaluation of area-under-the-concentration-time curve to time of maximum concentration of reference product, calculated for each subject (AUC $C_{\text {Reftmax }}$ )

randomly selected. Thus it would be expected that the results apply to an important portion of drug products on the Saudi market. 4) Although Saudi Arabia's BE regulations are very similar to most $\mathrm{BE}$ regulations worldwide, our results may not apply to similar drugs on other national markets. 5) Our study was not designed to partition intrasubject variability into its various components. Thus, it is not clear how much of the observed intra-subject variability is related to the generic products themselves (generic product quality variability or subject-by-product variability) and how much to methodological issues. 6) We observed significant (unadjusted) period and sequence effects in 6 and 2 of the 14 studies, respectively. It is likely that the apparent significance is due in large part to multiple comparisons and relatively large sample sizes, since we have also observed significant product effect in 8 of the 14 studies. The presence of period or sequence effect doesn't influence BE conclusions. Sequence effect and period effect may indicate unequal carryover, which is not likely given the length of the washout periods and the fact that baseline drug concentrations were undetectable in all periods for all 14 drugs. Sequence effect may also indicate that the groups (the 4 sequences) are different, which is also not likely because of randomization. However, it may also be due to product-by- period effect, which cannot be rolled out. Finally, period effect may indicate temporal changes, such as changes in patients' comfort level, familiarization with study, compliance, venous access, and drug stability. The latter is not likely because analysis of all drugs was performed well within each drug's pre-established stability period. 7) We have loss of follow up for one or more periods in 13 of the 14 studies, however, this resulted in negligible imbalance among the 4 sequences and negligible loss of power. 8) Finally, in retrospect, few of the 14 studies did not have adequate power to show $\mathrm{BE}$ for $\mathrm{C}_{\max }$, however, this would strengthen the main conclusions of the study.

\section{Conclusions}

Based on studying 42 randomly-selected generic products of 14 immediate-release, non-combinational, oral drugs with the highest number of generic products on the Saudi market, we can conclude that: 1) On-market generic products continue to be reference-bioequivalent. 2) Referencebioequivalent generic products are bioequivalent to each other, despite the presence of some generic-reference deviations that are $>6$ percentage points. 3) Referencegeneric and generic-generic average deviations are small (on average 3-5 percentage points) and similar. 4) 
Reference-generic and generic-generic $\mathrm{C}_{\max }$ intra-subject variations are large, similar, and can be present despite fulfilment of average BE criteria. However, they may be mostly related to methodological factors. 5) Average $\mathrm{BE}$ testing using $\mathrm{AUC}_{\mathrm{T}}, \mathrm{AUC}_{\mathrm{I}}$, and $\mathrm{C}_{\max }$ is insensitive to variability in $\mathrm{T}_{\max }$ and $A U C_{\text {Reftmax }}$. However, the intra-subject variabilities of the two parameters are similar, do not depend on whether a generic product is compared to a reference product or to another generic product, and may not be due to inter-product differences; suggesting limited usefulness of $\mathrm{T}_{\max }$ and $\mathrm{AUC}_{\text {Reftmax }}$ in $\mathrm{BE}$ evaluation. 6) $\mathrm{AUC}_{72}$ appears as informative as $\mathrm{AUC}_{\mathrm{T}}$ for drugs with long plasma half-life.

We believe that the study is the most rigorous study of on-market, generic drug products. It provided strong supporting evidence of the post-marketing quality and interchangeability of generic products and of the adequacy of current BE standards. It should allay fears of healthcare professionals and patients about the use of generic products, whether in the form of generic substitution or reference-to-generic or generic-to-generic switching.

\section{Additional files}

Additional file 1: Table S1. Blood sampling schedule of 14 bioequivalence studies on 14 immediate-release, non-combinational, oral drugs. (DOCX $14 \mathrm{~kb}$ )

Additional file 2: Table S2. Estimated and actual intra-subject CV of 14 bioequivalence studies on 14 immediate-release, non-combinational, oral drugs. (DOCX $41 \mathrm{~kb})$

Additional file 3: Table S3. Characteristics of three randomly-selected generic products and the reference product of 14 immediate-release, non-combinational, oral drugs. (DOCX $27 \mathrm{~kb}$ )

Additional file 4: Figure S1. Concentration-time curves of a reference and three randomly-selected generic products of 14 immediate-release, non-combinational, oral drugs. Concentration-time curves of a reference and three randomly-selected generic products of 14 immediate-release, non-combinational, oral drugs (a to n). Data represent mean concentrations. Blue diamond indicates reference, red square generic a, green triangle generic b, and purple cross generic c. (PPTX $30561 \mathrm{~kb}$ )

Additional file 5: Figure S2. Log-concentration-time curves of a reference and three randomly-selected generic products of 14 immediate-release, non-combinational, oral drugs. Log-concentration-time curves of a reference and three randomly-selected generic products of 14 immediate-releases, non-combinational, oral drugs (a to n). Data represent mean log-transformed concentrations. Blue diamond indicates reference, red square generic a, green triangle generic b, and purple cross generic c. (PPTX $30562 \mathrm{~kb}$ )

Additional file 6: Table S4. Main pharmacokinetic parameters of three randomly-selected generic products and reference product of 14 immediate-release, non-combinational, oral drugs. (DOCX 40 kb)

Additional file 7: Table S5. Analysis of variance of 14 bioequivalence studies on 14 immediate-release, non-combinational, oral drugs. (DOCX 15 kb)

Additional file 8: Table S6. Average bioequivalence of $A \cup C_{\text {Reftmax }}$ among three randomly-selected generic products and the reference product of 14 immediate-release, non-combinational, oral drugs. (DOCX $21 \mathrm{~kb}$ )

Additional file 9: Table S7. Average bioequivalence of 72-h-truncated area-under-the-concentration-time curve among three randomly-selected generic products and reference product of 2 immediate-release, noncombinational, oral, long half-life drugs. (DOCX 16 kb)

\section{Abbreviations}

ANOVA: Analysis of variance; AUC: Area-under-the-concentration-time-curve; $\mathrm{AUC}_{72}$ : Area-under-the-concentration-time-curve truncated to $72 \mathrm{~h}$; AUC: : Area-under-the-concentration-time-curve extrapolated to infinity; $A \cup C_{\text {Reftmax: }}$ Area-under-the-concentration-time-curve to time of maximum concentration $\left(T_{\max }\right)$ of reference product, calculated for each subject; $A \cup C_{T}$ : Area-under-the-concentration-time-curve to last measured concentration; BE: Bioequivalence; BMI: Body mass index; Cl: Confidence interval; $C_{\text {max: }}$ Maximum concentration; CV: Coefficient of variation (standard deviation/mean); FDA: Food and Drug Administration; Ga: Generic product a; Gb: Generic product b; Gc: Generic product c; HPLC: High performance liquid chromatography; KFSH\&RC: King Faisal Specialist Hospital and Research Center; LC-MS: Liquid chromatography-mass spectrometry; MSR: Mean square residual; NTI: Narrow therapeutic index; R: Reference product; $\mathrm{SD}$ : Standard deviation; $\mathrm{t}_{1 / 2}$ : Terminal elimination half-life; $T_{\max }$ : Time of maximum concentration; $\lambda$ : Apparent first-order elimination rate constant

\section{Acknowledgments}

Special thanks to participants of the study for their dedication and contribution to research. The superb assistance by the research staff at the Clinical Studies and Empirical Ethics Department, namely, Faduma A Farah, Faduma S Shire, Ahmed Yusuf, Saleh Al-Dghither, is gratefully acknowledged.

\section{Funding}

This study was funded by a research grant to $\mathrm{MMH}$ from the King Abdul-Aziz City for Science and Technology (KACST), under National Comprehensive Plan for Science and Technology, Riyadh, Saudi Arabia (10-Bio961-20).

\section{Availability of data and materials}

The dataset supporting the conclusions of this article is available upon request from $\mathrm{MMH}$ and in the attached Additional Files.

\section{Role of the funder/sponsor}

The funder had no role in the design and conduct of the study; in the collection, management, analysis, and interpretation of the data; or in the preparation, review, or approval of the manuscript.

\section{Authors' contributions}

$\mathrm{MMH}$ had full access to all of the data in the study and takes responsibility for the integrity of the data and the accuracy of the data analysis. Study concept and design: MMH and EAG. Acquisition of data: SP, EAG, NAK. Analysis, or interpretation of data: $\mathrm{MMH}$. Drug concentration measurements: $\mathrm{RH}, \mathrm{RA}, \mathrm{NB}$, SNA. Statistical analysis: MMH. Manuscript writing: $\mathrm{MMH}$. Critical revision of the manuscript for important intellectual content: all authors. All authors have approved the final version of the manuscript and agreed to be accountable to all aspects of the work.

\section{Ethics approval and consent to participate}

The study was conducted according to the ethical guidelines of the Declaration of Helsinki and was approved by the King Faisal Specialist Hospital \& Research Center's Research Ethics Committee (RAC 2101100). All participants provided a written informed consent.

\section{Consent for publication}

Not applicable.

\section{Competing interests}

The authors declare that they have no competing interests.

\section{Publisher's Note}

Springer Nature remains neutral with regard to jurisdictional claims in published maps and institutional affiliations.

Received: 21 June 2017 Accepted: 22 November 2017

Published online: 08 December 2017

\section{References}

1. World Health Organization. Access to new medicines in Europe: technical review of policy initiatives and opportunities for collaboration and research 2015. http:// www.euro.who.int/en/health-topics/Health-systems/health-technologies-andmedicines/publications/2015/access-to-new-medicines-in-europe-technical- 
review-of-policy-initiatives-and-opportunities-for-collaboration-and-research-2015. Accessed June 12, 2017

2. Toverud E-L, Hartmann K, Håkonsen H. A systematic review of physicians' and pharmacists' perspectives on generic drug use: what are the global challenges? Appl Health Econ Health Policy. 2015;13(Suppl 1):35-45.

3. Dunne SS, Dunne CP. What do people really think of generic medicines? A systematic review and critical appraisal of literature on stakeholder perceptions of generic drugs. BMC Med. 2015;13:173.

4. Hassali MA, Alrasheedy AA, McLachlan A, Nguyen TA, AL-Tamimi SK, Ibrahim MIM, Aljadheye $\mathrm{H}$. The experiences of implementing generic medicine policy in eight countries: a review and recommendations for a successful promotion of generic medicine use. Saudi Pharm J. 2014;22(6):491-503.

5. O'Leary A, Usher C, Lynch M, Hall M, Hemeryk L, Spillane S, Gallagher P, Barry M. Generic medicines and generic substitution: contrasting perspectives of stakeholders in Ireland. BMC Res Notes. 2015;8:790.

6. Kumar R, Hassali MA, Saleem F, Alrasheedy AA, Kaur N, Wong ZY, Abdul Kader MASK. Knowledge and perceptions of physicians from private medical centres towards generic medicines: a nationwide survey from Malaysia. J Pharm Policy Pract. 2015;8(1):11.

7. Davit B, Braddy AC, Conner DP, Yu LX. International guidelines for bioequivalence of systemically available orally administered generic drug products: a survey of similarities and differences. APPS J. 2013;15(4):974-90.

8. Chen M-L, Shah VP, Crommelin DJ, Shargel L, Bashaw D, Bhatti M, Blume H, Dressman J, Ducharme M, Fackler P, Hyslop T, Lutter L, Morais J, Ormsby E, Thomas S, Tsang YC, Velagapudi R, Yu LX. Harmonization of regulatory approaches for evaluating therapeutic equivalence and interchangeability of multisource drug products: workshop summary report. AAPS J. 2011;13(4):556-64.

9. Kaushal N, Singh SK, Gulati M, Vaidya Y, Kaushik M. Study of regulatory requirements for the conduct of bioequivalence studies in US, Europe, Canada, India, ASEAN and SADC countries: impact on generic drug substitution. J App Pharm Sci. 2016;6(4):206-22.

10. Statistical approaches to establishing bioequivalence. US DHHS, FDA, CDER 2001. https://www.fda.gov/downloads/drugs/guidances/ucm070244.pdf. Accessed June 12, 2017.

11. Dunne S, Shannon B, Dunne C, Cullen W. A review of the differences and similarities between generic drugs and their originator counterparts, including economic benefits associated with usage of generic medicines, using Ireland as a case study. BMC Pharmacol Toxicol. 2013;14:1

12. Hendeles I, Hochhaus G, Kazerounian S. Generic and alternative brand-name pharmaceutical equivalents: select with caution. Am J Hosp Pharm. 1993; 50(2):323-9

13. US Food and Drug Administration (FDA). Review of therapeutic equivalence generic Bupropion XL 300 mg and Wellbutrin XL 300 mg. Silver Spring: FDA; 2013. https:/mww.fda.gov/aboutfda/centersoffices/officeofmedicalproductsandtobacco/ cder/ucm153270.htm. Accessed June12, 2017

14. US Food and Drug Administration (FDA). Methylphenidate hydrochloride extended release tablets (generic Concerta) made by Mallinckrodt and Kudco. Silver Spring: FDA; 2014. https://www.fda.gov/Drugs/DrugSafety/ucm422568. htm. Accessed June 12, 2017

15. Gasser UE, Fischer A, Timmermans JP, Arnet I. Pharmaceutical quality of seven generic Levodopa/Benserazide products compared with original Madopar ${ }^{\circledast} /$ Prolopa ${ }^{\circledast}$. BMC Pharmacol Toxicol. 2013;14:24.

16. Del Tacca M, Pasqualetti G, Di Paolo A, Virdis A, Massimetti G, Gori G, Versari D, Taddei S, Blandizzi C. Lack of pharmacokinetic bioequivalence between generic and branded amoxicillin formulations. A post-marketing clinical study on healthy volunteers. Br J Clin Pharmacol. 2009;68(1):34-42.

17. Ting TY, Jiang $W$, Lionberger $R$, Wong J, Jones JW, Kane MA, Krumholz A, Temple R, Polli JE. Generic lamotrigine versus brand-name Lamictal bioequivalence in patients with epilepsy: a field test of the FDA bioequivalence standard. Epilepsia. 2015;56(9):1415-24.

18. Kesselheim AS, Misono AS, Lee JL, Stedman MR, Brookhart MA, Choudhry NK, Shrank WH. Clinical equivalence of generic and brand-name drugs used in cardiovascular disease. A systematic review and meta-analysis. JAMA. 2008:300(21):2514-26.

19. Manzoli L, Flacco ME, Boccia S, D'Andrea E, Panic N, Marzuillo C, Siliquini R, Ricciardi W, Villari P, loannidis JPA. Generic versus brand-name drugs used in cardiovascular diseases. Eur J Epidemiol. 2016;31:351-68.

20. Kesselheim AS, Stedman MR, Bubrick EJ, Gagne JJ, Misono AS, Lee JL, Brookhart MA, Avorn J, Shrank WH. Seizure outcomes following use of generic vs. brand-name antiepileptic drugs: a systematic review and metaanalysis. Drugs. 2010;70(5):605-21.
21. Singh AK, Narsipur SS. Cyclosporine: a commentary on brand versus generic formulation exchange. J Transp Secur. 2011;2011:480642.

22. Montoya-Eguía SL, Garza-Ocañas L, Badillo-Castañeda CT, Tamez-de la OE, Zanatta-Calderón T, Gómez-Meza MV, Garza-Ulloa H. Comparative pharmacokinetic study among 3 metformin formulations in healthy Mexican volunteers: a single-dose, randomized, open-label, 3-period crossover study. Curr Therap Res. 2015;77:18-23.

23. Anderson S, Hauck WW. The transitivity of bioequivalence testing: potential for drift. Int J Clin Pharm and Therap. 1996;34:369-74.

24. Johnston A. Equivalence and interchangeability of narrow therapeutic index drugs in organ transplantation. Eur J Hosp Pharm SciPract. 2013;20(5):302-7.

25. Yim D-S. Simulation of the AUC changes after generic substitution in patients. J Korean Med Sci. 2009;24(1):7-12.

26. Maliepaard M, Banishki N, Gispen-de Wied CC, Teerenstra S, Elferink AJ. Interchangeability of generic anti-epileptic drugs: a quantitative analysis of topiramate and gabapentin. Eur J Clin Pharmacol. 2011;67(10):1007-16.

27. Yu Y, Teerenstra S, Neef C, Burger D, Maliepaard M. Investigation into the interchangeability of generic formulations using immunosuppressants and a broad selection of medicines. Eur J Clin Pharmacol. 2015;71(8):979-90.

28. Krauss GL, Caffo B, Chang YT, Hendrix CW, Chuang K. Assessing bioequivalence of generic antiepilepsy drugs. Ann Neurol. 2011;70(2):221-8. doi:10.1002/ana.22452

29. Friesen MH, Walker SE. Are the current bioequivalence standards sufficient for the acceptance of narrow therapeutic index drugs? Utilization of a computer simulated warfarin bioequivalence model. J Pharm Pharmaceut Sci. 1999;2(1):15-22.

30. Karalis V, Bialer M, Macheras P. Quantitative assessment of the switchability of generic products. Eur J Pharm Sci. 2013;50(3-4):476-83.

31. Privitera MD, Welty TE, Gidal BE, Diaz FJ, Krebill R, Szaflarski JP, Dworetzky BA, Pollard JR, Elder EJ Jr, Jiang W, Jiang X, Berg M. Generic-to-generic lamotrigine switches in people with epilepsy: the randomised controlled EQUIGEN trial. Lancet Neurol. 2016:15(4):365-72. doi:10.1016/S1474-4422(16)00014-4.

32. Atif M, Azeem M, Sarwar MR. Potential problems and recommendations regarding substitution of generic antiepileptic drugs: a systematic review of literature. Spring. 2016;5:182.

33. Vercaigni LM, Zhanel GG. Clinical significance of bioequivalence and interchangeability of narrow therapeutic range drugs: focus on warfarins. J Pharm Pharmaceut Sci. 1998;1(3):92-4.

34. Reiffel JA, Kowey PR. Generic antiarrythmics are not therapeutically equivalent for the treatment of tachyarrhythmias. Am J Cardiology. 2000;85:1151-3.

35. Hsuan FC. Some statistical considerations on the FDA draft guidance for individual bioequivalence. Stat Med. 2000;19(20):2879-84.

36. Vossler DG, Anderson GD, Bainbridge J. AES position statement on generic substitution of antiepileptic drugs. Epilepsy Curr. 2016;16(3):209-11. doi:10. 5698/1535-7511-16.3.209.

37. Perucca E. The safety of generic substitution in epilepsy. Lancet Neurol. 2016; 15(4):344-5. doi:10.1016/S1474-4422(16)00042-9.

38. Alrasheedy AA, Hassali MA, Aljadhey H, Ibrahim MI, Al-Tamimi SK. Is there a need for a formulary of clinically interchangeable medicines to guide generic substitution in Saudi Arabia? J Young Pharm. 2013;5(2):73-5. doi:10.1016/j.jyp. 2013.06.006.

39. Salhia HO, Ali A, Rezk NL, El Metwallya A. Perception and attitude of physicians toward local generic medicines in Saudi Arabia: a questionnaire-based study. Saudi Pharm J. 2015;23(4):397-404.

40. Dickert N, Grady C. What's the price for a research subject? Approaches to payment for research participation. N Engl J Med. 1999;341(3):198-203.

41. Hussein R, Hammami MM. Fully validated diclofenac HPLC assay. ACAIJ. 2009;8(2):124-9.

42. Al-Dgither S, Yusuf A, Hammami MM. Fluconazole: stability and analysis in human plasma by simple high performance liquid chromatography. FABAD J Pharm Sci. 2011:34:179-86.

43. Alvi SN, Yusuf A, Al Gaai E, Hammami MM. Rapid determination of ibuprofen concentration in human plasma by high performance liquid chromatography. WJPPS. 2014;3(8):1767-77.

44. Al-Swayeh R, Alvi SN, Hammami MM. A validated HPLC method for the determination of ranitidine in human plasma: application to bioavailability studies. ACAIJ. 2015;15(8):339-44.

45. Hussein RF, Hammami MM. Determination of cephalexin level and stability in human plasma by fully validated rapid HPLC analysis. WJPPS. 2014;3(12):20-31. 
46. Binhashim NH, Alvi SN, Hammami MM. A validated reversed phase HPLC assay for the determination of metronidazole in human plasma. WJPPS. 2014;3(12):32-41.

47. Yusuf A, Alvi SN, Hammami MM. Development and validation of RP-HPLC method for the determination of metformin in human plasma. WJPPS. 2015; 4(7):128-38.

48. Alswayeh R, Alvi SN, Hammami MM. Rapid determination of amoxicillin level in human plasma by high performance liquid chromatography. WJPPS. 2015:4(7):1657-67.

49. Yusuf A, Alvi SN, Hammami MM. Development and validation of RP HPLC method for the determination of atenolol in human plasma. WJPPS. 2016; 5(2):169-79.

50. Alswayeh R, Hussein RF, Alvi SN, Hammami MM. Rapid determination of ciprofloxacin concentration in human plasma by high performance liquid chromatography. WJPPS. 2016:5(3):1765-74.

51. Hussein RF, Binhashim NH, Alvi SN, Hammami MM. A validated reversed phase HPLC assay for the determination of omeprazole in human plasma. EJPMR. 2016;3(6):26-30

52. Alswayeh R, Alvi SN, Hammami MM. Rapid determination of acetaminophen levels in human plasma by high performance liquid chromatography. A J Pharmtech Res. 2016;6(3):710-9.

53. Alvi SN, Al Dgither S, Hammami MM. Rapid determination of clarithromycin in human plasma by LCMS/MS assay. Pharm Anal Chem Open Access. 2016;2:1.

54. Alvi SN, Hussein RF, Al Dgither S, Hammami MM. Quantitation of amlodipine in human plasma by LCMS/MS assay. Int J Pharm Pharm Sci. 2016;8(8):268-72.

55. Randomization.com. Dallal GE. http://www.randomization.com/. Accessed June 12, 2017.

56. FARTSSIE. David Dubins D. http://individual.utoronto.ca/ddubins. Accessed June12, 2017

57. Jiang W, Makhlouf F, Schuirmann DJ, Zhang X, Zheng N, Conner D, Yu LX, Lionberger R. A bioequivalence approach for generic narrow therapeutic index drugs: evaluation of the reference-scaled approach and variability comparison criterion. AAPS J. 2015;17(4):891-901

58. Hammami MM, Yusuf A, Shire FS, Hussein R, Al-Swayeh R. Does the placebo effect modulate drug bioavailability? Randomized cross-over studies of three drugs. JNCT. 2017;201716:10. doi:10.1186/s12952-017-0075-2.

59. Henney J. Review of generic bioequivalence studies. JAMA. 1999;282(21):1995.

60. Davit BM, Nwakama PE, Buehler GJ, Conner DP, Haider SH, Patel DT, Yang Y, Yu LX, Woodcock J. Comparing generic and innovator drugs: a review of 12 years of bioequivalence data from the United States Food and Drug Administration. Ann Pharmacother. 2009;43(10):1583-97.

61. Espay AJ, Norris MM, Eliassen JC, Dwivedi A, Smith MS, Banks C, Allendorfer $J B$, Lang AE, Fleck DE, Linke MJ, Szaflarski JP. Placebo effect of medication cost in Parkinson disease: a randomized double-blind study. Neurology. 2015:84:794-802

62. Thakkar KB, Billa G. The concept of generic drugs and patented drugs vs. brand name drugs and non-proprietary (generic) name drugs. Front Pharmacol. 2013:4:113.

63. Piguet V, D'Incau S, Besson M, Desmeules J, Cedraschi C. Prescribing generic medication in chronic musculoskeletal pain patients: an issue of representations, trust, and experience in a Swiss cohort. PLoS One. 2015; 10(8):e0134661.

64. Tóthfalusi L, Endrényi L,S-C. Statistical and regulatory considerations in assessments of interchangeability of biological drug products. Eur J Health Econ. 2014;15(Suppl 1):5-11.

65. Hammami MM, Alvi SN. Large intra-subject variability in caffeine pharmacokinetics: randomized cross-over study of single caffeine product. Drug Res. 2017;67:1-8. doi:10.1055/s-0043-110144.

\section{Submit your next manuscript to BioMed Central and we will help you at every step:}

- We accept pre-submission inquiries

- Our selector tool helps you to find the most relevant journal

- We provide round the clock customer support

- Convenient online submission

- Thorough peer review

- Inclusion in PubMed and all major indexing services

- Maximum visibility for your research

Submit your manuscript at www.biomedcentral.com/submit
) Biomed Central 